\title{
Non-Definite Sturm-Liouville Problems with a Weight Function having Two Turning Points
}

\author{
by \\ Mervis Kikonko, \\ B.Arts(with Education), University of Zambia, 2008 \\ A thesis submitted to \\ the Faculty of Graduate Studies and Postdoctoral Affairs \\ in partial fulfillment of \\ the requirements for the degree of \\ Master of Science
}

School of Mathematics and Statistics

Ottawa-Carleton Institute for Mathematics and Statistics

Carleton University

Ottawa, Ontario, Canada

(C) Copyright 2011, Mervis Kikonko 


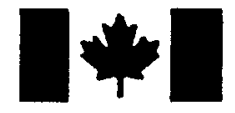

\author{
Library and Archives \\ Canada \\ Published Heritage \\ Branch \\ 395 Wellington Street \\ Ottawa ON K1A ON4 \\ Canada
}

Bibliothèque et

Archives Canada

Direction du

Patrimoine de l'édition

395 , rue Wellington

Ottawa ON K1A ON4

Canada
Your file Votre référence
ISBN: 978-0-494-79604-7
Our file Notre référence
ISBN: $978-0-494-79604-7$
NOTICE:

The author has granted a nonexclusive license allowing Library and Archives Canada to reproduce, publish, archive, preserve, conserve, communicate to the public by telecommunication or on the Internet, loan, distribute and sell theses worldwide, for commercial or noncommercial purposes, in microform, paper, electronic and/or any other formats.

The author retains copyright ownership and moral rights in this thesis. Neither the thesis nor substantial extracts from it may be printed or otherwise reproduced without the author's permission.
AVIS:

L'auteur a accordé une licence non exclusive permettant à la Bibliothèque et Archives Canada de reproduire, publier, archiver, sauvegarder, conserver, transmettre au public par télécommunication ou par l'Internet, prêter, distribuer et vendre des thèses partout dans le monde, à des fins commerciales ou autres, sur support microforme, papier, électronique et/ou autres formats.

L'auteur conserve la propriété du droit d'auteur et des droits moraux qui protège cette thèse. $\mathrm{Ni}$ la thèse ni des extraits substantiels de celle-ci ne doivent être imprimés ou autrement reproduits sans son autorisation.
In compliance with the Canadian Privacy Act some supporting forms may have been removed from this thesis.

While these forms may be included in the document page count, their removal does not represent any loss of content from the thesis.
Conformément à la loi canadienne sur la protection de la vie privée, quelques formulaires secondaires ont été enlevés de cette thèse.

Bien que ces formulaires aient inclus dans la pagination, il n'y aura aucun contenu manquant.

\section{Canadä}




\section{Abstract}

We study the non-definite Sturm-Liouville problem with a weight function having two turning points on a finite closed interval. We find the piecewise smooth solution over the closed interval and give the dispersion relation for the eigenvalues. We then solve this dispersion relation numerically using Maple software in order to calculate a great many eigenvalues. We then find the piecewise smooth eigenfunctions associated with each of the eigenvalues and verify various theorems from Sturm-Liouville Theory and their extensions to indefinite/nondefinite cases. We also extend key results of Atkinson and Jabon [7] and give estimates on the Richardson numbers of specific Sturm-Liouville problems having two turning points. We then present graphs of some of the eigenfunctions to check the oscillation numbers of the eigenvalues associated with these functions. 


\section{Acknowledgements}

I should like to express my special thanks to the School of Mathematics and Statistics at Carleton University, NSERC and my thesis supervisor Dr. Angelo B. Mingarelli for their generous funding towards the completion of this research. Specifically, I thank my supervisor for suggesting my thesis topic and for being there for me whenever I needed help with my research. Working under his supervision was a pleasure and a good experience from which I learnt a lot, academically. I acknowledge with thanks comments by members of my defense committee which included Professor Oakham, Dr. Amundsen and Dr. Smith.

Special thanks to Prof David Theo from the Department of Mathematics and Statistics at the University of Zambia for suggesting my name to be the first recipient of the Anglin Zambia Scholarship which was the main source of income for my studies. Thanks too to Dr. Victor Nawa of the same department for suggesting the research area for my studies.

I must thank all the people who supported me during my studies in Canada, most importantly Douglas and Mary Anglin who made my stay here possible by being awarded the inaugural Anglin Scholarship. My sincere thanks to my husband Edgar Shamalambo for his love and care for our children in my long absence as I undertook my studies. I thank my children Edgar, Eric and Nelly for being so strong as to remain at home without a mother for more than two years. My sincere thanks to my parents and friends for the moral support they gave me during my research phase and the writing of my thesis. Finally, I also wish to express my thanks to all the people who came into my life and with whom we shared wonderful moments during my stay in Canada over the past two years. 


\section{Contents}

$\begin{array}{ll}\text { Abstract } & \text { i }\end{array}$

$\begin{array}{ll}\text { Acknowledgements } & \text { ii }\end{array}$

List of figures $\quad v$

1 Introduction 1

2 Definitions and Preliminary Results 4

2.1 The Prüfer system . . . . . . . . . . . . . . 7

2.2 Comparison Theorems $\ldots \ldots \ldots \ldots \ldots \ldots$

3 The Non-Definite Sturm-Liouville Problem $\quad 20$

3.1 Estimating the Richardson Numbers $\ldots \ldots \ldots \ldots$

3.2 The Nature of the Spectrum . . . . . . . . . . . . . . . . . . 34

4 Explicit Solution of the Non-Definite Problem 36 
5 Discussion

6 Conclusion 


\section{List of Figures}

2.1 The minimum of $u(x)$ in this figure is not possible because the corresponding slope $\theta^{\prime}(x)$ at that point would have to be negative, violating inequality (2.8) . 11

2.2 Graph of $\sin (4 x) \ldots \ldots \ldots \ldots \ldots \ldots \ldots \ldots$

2.3 Graphs of the first four eigenfunctions of problem in Example 2.2.10 . . . . 18

3.1 Graphs of a solution $u\left(x, \lambda_{1}\right) \ldots \ldots \ldots \ldots \ldots \ldots$

3.2 A typical solution $u(x)$ of $(3.1)$ on $[-1,2]$ for large $\lambda>0 \ldots \ldots 25$

4.1 Graphs of some eigenfunctions of $(3.1)-(3.2)$ for the case $q_{0}=\pi^{2} \ldots \ldots 38$

4.2 Graphs of the real and imaginary part of the eigenfunction corresponding to $\lambda=3.2465+5.6334 i$ when $q_{0}=\pi^{2} \ldots \ldots \ldots \ldots \ldots \ldots \ldots$

4.3 Graphs of some eigenfunctions of $(3.1)-(3.2)$ for the case $q_{0}=6 \pi^{2} \quad \ldots \quad 39$

4.4 Graphs of some eigenfunctions of $(3.1)-(3.2)$ for the case $q_{0}=6 \pi^{2}$ and $\lambda=-6.4344-14.4314 i \ldots \ldots \ldots \ldots \ldots \ldots \ldots$

4.5 Graphs of some eigenfunctions of $(3.1)-(3.2)$ for the case $q_{0}=6 \pi^{2}$ and $\lambda=-13.4034-13.5248 i \ldots \ldots \ldots \ldots \ldots \ldots \ldots \ldots \ldots \ldots$

4.6 Graphs of some eigenfunctions of $(3.1)-(3.2)$ for the case $q_{0}=6 \pi^{2}$ and

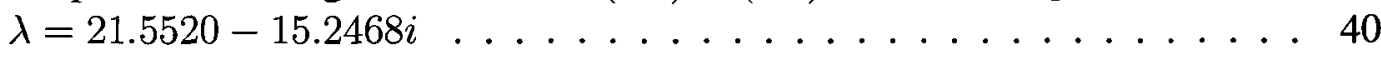


4.7 Graphs of some eigenfunctions of $(3.1)-(3.2)$ for the case $q_{0}=6 \pi^{2}$ and $\lambda=52.0258+7.0997 i \ldots \ldots \ldots 1$ 


\section{Chapter 1}

\section{Introduction}

The Sturm-Liouville equation, named after Jacques Charles François Sturm (1803-1855) and Joseph Liouville (1809-1882) is a real second-order linear differential equation of the form

$$
-\left(p(x) u^{\prime}(x)\right)^{\prime}+q(x) u(x)=\lambda w(x) u(x)
$$

on the bounded or unbounded interval $(a, b)$, the endpoints $a$ and $b$ being finite or infinite, and $u$ is a function of the independent variable $x$. The coefficient functions $p(x)$ and $q(x)$ and the weight function $w(x)$ are assumed to be real-valued, with $p(x)>0$. To ensure existence of solutions and for simplicity, we take it that the functions $q(x)$ and $w(x)$ are continuous or piecewise continuous with $p(x)$ being continuously differentiable (of class $C^{1}(a, b)$ ). More generally, they satisfy the integrability conditions

$$
p^{-1}, q, w, \in L_{l o c}(a, b) ;
$$

that is, $p^{-1}, q$, and $w$ have well-defined and finite Lebesgue integrals on all compact subintervals of $(a, b)$. In the case of a regular problem the solution $u$ is required to satisfy the separated homogeneous boundary conditions:

$$
\begin{gathered}
\alpha_{1} u(a)+\alpha_{2} p(a) u^{\prime}(a)=0 \\
\beta_{1} u(b)+\beta_{2} p(b) u^{\prime}(b)=0
\end{gathered}
$$

where $\alpha_{1}$ and $\alpha_{2}$ are not both zero; similarly for $\beta_{1}$ and $\beta_{2}$.

The value of the generally complex parameter $\lambda$ is not specified in the equation. Finding the values of $\lambda$ for which there exists a non-trivial solution satisfying both the equation and the boundary conditions is part of the problem called the Sturm-Liouville eigenvalue problem. Such values of $\lambda$ when they exist are called the eigenvalues of the boundary value problem 
defined by (1.1) and the prescribed set of boundary conditions (1.2)-(1.3). The corresponding solutions (for such a $\lambda$ ) are called the eigenfunctions of this problem. Each eigenvalue may have one or more eigenfunctions corresponding to it, depending on the boundary conditions. When an eigenvalue has only one eigenfunction, then it is called a simple eigenvalue, otherwise it is non-simple. The set of all the eigenvalues of a regular Sturm-Liouville problem is called the spectrum of the problem. By a solution, we mean a generally complex-valued function $u(x)$ of the real variable $x$ such that $u$ and $p u^{\prime}$ are absolutely continuous on $[a, b]$ that satisfies the boundary conditions (1.2)-(1.3). Under the normal assumptions on the coefficient functions $p(x), q(x)$ and $w(x)$ above, they induce a Hermitian differential operator in some function space defined by the boundary conditions. The theory of the existence and asymptotic behavior of the eigenvalues as the oscillation number tends to infinity, the corresponding qualitative theory of the eigenfunctions and their completeness in a suitable function space makes up what is commonly known as Sturm-Liouville (S-L) theory.

This theory is important in applied mathematics, where SL problems occur very commonly. The differential equations considered here arise directly as mathematical models of motion according to Newton's law, but more often as a result of using the method of separation of variables to solve the classical partial differential equations of physics, such as Laplace's equation, the heat equation, and the wave equation. Sturm-Liouville problems have been discovered as describing the mathematics underlying a variety of physical phenomena. Thus, they have been applied in various fields of study like Engineering and Physics. According to Hinton and Schaefer [3], their use in problems of vibrations, heat transfer, quantum mechanics and a host of other areas have proven successful for many years.

Much has been written about Sturm-Liouville problems since the work of Sturm and Liouville in the 19th century. As Hinton and Schaefer [3] put it, in the period 1836-38, Sturm and Liouville published a remarkable set of papers which initiated the subject. This led to what is now called the qualitative theory of differential equations. They go on to say that Sturm was mainly concerned with the qualitative behavior of the eigenfunctions, while Liouville was more concerned with the eigenfunction expansions. As a result, in Sturm-Liouville theory, there are two basic problems:

1. To establish the existence of eigenvalues and eigenfunctions and describe them qualitatively, and to some extent, quantitatively.

2. To prove the existence of some function space each of whose functions can be expressed as an infinite series of eigenfunctions.

Our discussions in this thesis will only involve taking up the first problem. Thus, we study the regular Sturm-Liouville problem in which the weight function $w(x)$ changes sign on $[a, b]$. The main motivation for this paper is one of the pioneering articles on indefinite 
Sturm-Liouville problems by Jabon and Atkinson [7]. Atkinson and Jabon considered the Sturm-Liouville problem in which the weight function changes sign once on a finite closed interval $[a, b]$. They considered a specific problem which seemed to give some insight into the general case. They obtained bounds for the so-called Richardson numbers (defined in Chapter 2) and presented results on the spectrum of a specific problem. We extend their results to a Sturm-Liouville problem in which the weight function changes sign twice on the finite interval $[a, b]$. Such problems, together with the self-adjoint boundary conditions above, are called indefinite (more specifically, non-definite Sturm-Liouville problems.) The values of $x$ about which $w(x)$ changes signs are called turning points.

Chapter 2 reviews the Hilbert space $L^{2}[a, b]$ and its subspaces $C^{n}[a, b]$. We give a few definitions in relation to operators in Hilbert space, we state and/or prove various theorems, lemmas and propositions. Furthermore the Prüfer transformation which is key in the study of various properties of Sturm-Liouville problems is discussed. In Chapter 3 we discuss the Sturm-Liouville problem in which the weight function $w(x)$ changes sign twice on a specific interval, $[-1,2]$, while verifying some results of Chapter 2 . It is discovered that some of the properties enjoyed by regular Sturm-Liouville problems are not so evident when applied to the problem at hand. We also obtain bounds for the so-called Richardson numbers for the non-definite problem. In the first place, we discuss a good number of results given in [7] as there seems to be many typographic and other errors in some statements and proofs in [7]. After fixing these, the results are then extended to the non-definite problem given in Chapter 3 so that we may deduce what happens when the weight function changes sign twice on the finite interval.

In Chapter 4 the solution to the non-definite problem is given together with the dispersion relation which is then solved in order to estimate the eigenvalues and so the eigenfunctions. Due to the complicated nature of the problem we see that it is very difficult to write down the whole sequence of eigenvalues (in addition, the dispersion relation cannot be solved exactly for its roots).

We present the graphs of various eigenfunctions from which we deduce the oscillation numbers (or number of zeros) of the eigenfunctions. We also display the effect of increasing or reducing the value of $q_{0}$ in relation to the eigenvalues and the corresponding oscillation numbers of eigenfunctions. Using the graphs of such eigenfunctions, we verify some properties that are obvious in the regular case and apparently new in the non-definite case. In Chapter 5 we discuss the results obtained in the preceding chapters and present our conclusions in Chapter 6. 


\section{Chapter 2}

\section{Definitions and Preliminary Results}

The following definitions are all given in [10]. A Hilbert space is an inner product space in which the inner product induces a norm under which the space is complete. The proof that $L^{2}[a, b]$ is a complete normed space is given in [10]. For simplicity, we shall denote $L^{2}[a, b]=H$. For the closed interval $[a, b]$, we shall denote the real or complex Hilbert space of all square-integrable functions by $L^{2}[a, b]$, with inner product

$$
(u, v)=\int_{a}^{b} u(x) \overline{v(x)} d x
$$

and norm

$$
\|u\|=(u, u)^{1 / 2}=\left(\int_{a}^{b}|u(x)|^{2} d x\right)^{1 / 2}
$$

We first give some definitions. The symbol $B(x, \varepsilon)$ refers to an abstract ball in a normed space having center $x$ and radius $\varepsilon$ defined by the set of all points $y$ such that $\|y-x\|<\varepsilon$.

Definition 2.0.1. (Open and closed sets)

A subset $S$ of a normed space $E$ is called open if for every $x \in S$ there exists $\varepsilon>0$ such that $B(x, \varepsilon) \subset S$. A subset $S$ is called closed if its complement is open.

Definition 2.0.2. (Closure)

Let $S$ be a subset of a normed space $E$. By the closure of $S$, denoted by $\bar{S}$, we mean the intersection of all closed sets containing $S$.

Definition 2.0.3. (Dense subset)

$A$ subset $S$ of a normed space $E$ is called dense in $E$ if $\bar{S}=E$. 
For each $n \geq 0$ let $C^{n}([a, b])$ be the subspace of all functions which possess continuous derivatives of orders $1,2, \ldots, n$ on $[a, b]$ and let $C^{\infty}[a, b]$ denote the subspace of all infinitely differentiable functions on $[a, b]$. We state without proof the following theorem and corollary stated in [14].

Theorem 2.0.4. The subspace of all continuous functions on $[a, b]$ is dense in $H$.

Corollary 2.0.5. The subspaces $C^{n}[a, b]$ and $C^{\infty}[a, b]$ are dense in $H$.

Definition 2.0.6. A linear operator on $H$ is a map $T$ with domain $D(T)$ and range $R(T)$ both contained in $H$ which satisfies the properties:

1. $D(T)$ is a subspace of $H$

2. $T(u+v)=T(u)+T(v)$, for all $u, v \in D(T)$

3. $T(\alpha u)=\alpha T(u)$, for all scalars $\alpha$ and for all $u \in D(T)$.

An operator $T$ is said to be densely defined in $H$ if $\overline{D(T)}=H$. For the definition of an adjoint operator, we adopt the definition given in [15].

Definition 2.0.7. Suppose $T$ is a densely defined linear operator in $H$ and let $D\left(T^{*}\right)$ denote the set of all $v \in H$ such that for some $v^{*} \in H$ we have

$$
(T u, v)=\left(u, v^{*}\right), \text { for all } u \in D(T) \text {. }
$$

Let $T^{*}: D\left(T^{*}\right) \rightarrow H$ denote the map defined by $T^{*} v=v^{*}$. Then $T^{*}$ is called the adjoint of $T$ so that (2.1) becomes

$$
(T u, v)=\left(u, T^{*} v\right), \text { for all } u \in D(T), v \in D\left(T^{*}\right)
$$

Definition 2.0.8. A densely defined linear operator $T$ on $H$ is said to be symmetric if

$$
(T u, v)=(u, T v), \text { for all } u, v \in D(T), \text { so } T \subseteq T^{*},
$$

i.e., $D(T) \subseteq D\left(T^{*}\right)$

$T$ is said to be self-adjoint if $T$ is symmetric and $D(T)=D\left(T^{*}\right)$, i.e., $T=T^{*}$. A symmetric linear operator for which there exists a real constant $\gamma$ such that $(T u, u) \geq \gamma(u, u)$ for all $u \in D(T)$ is said to be bounded below. In the special case where $(T u, u)>0$ for all $u \in D(T), T$ is said to be positive (definite). A negative (definite) linear operator is defined similarly. 
Definition 2.0.9. Disconjugacy

An $n^{\text {th }}$ order homogeneous linear differential equation

$$
L y=y^{(n)}+p_{1}(x) y^{(n)}+\cdots+p_{n}(x) y=0
$$

is called disconjugate on an interval I if no non-trivial solutions have $n$ zeros on I, multiple zeros being counted according to their multiplicity.

Definition 2.0.10. The Sturm-Liouville problem (1.1)-(1.3) is said to be regular if:

1. The end-points $a$ and $b$ are both finite,

2. The functions $p, q, w$ are defined on the closed interval $[a, b]$ to be continuous except for finitely many jumps, with $p, w$ strictly positive,

3. Boundary conditions of the form (1.2)-(1.3) are imposed at $a$ and $b$.

Definition 2.0.11. An eigenfunction $\varphi$ (real or not) for which

$$
(w \varphi, \varphi)=\int_{a}^{b} w|\varphi|^{2} d x=0
$$

is called a ghost state (see [17].)

In view of Definition 2.0.11, we state the following proposition.

Proposition 2.0.12. Any eigenfunction corresponding to a non-real eigenvalue of (1.1)-(1.3) is a ghost state.

Proof. First, we observe that non-real eigenvalues/eigenfunctions occur in complex-conjugate pairs, i.e., $\lambda$ is a non-real eigenvalue if and only if $\bar{\lambda}$ is an eigenvalue. Let $\bar{u}$ be an eigenfunction corresponding to a non-real eigenvalue, $\bar{\lambda}$. Then we get

$$
-\left(p(x) \bar{u}^{\prime}\right)^{\prime}+q(x) \bar{u}=\bar{\lambda} w(x) \bar{u} .
$$

Multiplying (1.1) by $\bar{u}$ and (2.2) by $u$ gives,

$$
-\left(p(x) u^{\prime}\right)^{\prime} \bar{u}+q(x)|u|^{2}=\lambda w(x)|u|^{2}
$$

and

$$
-\left(p(x) \bar{u}^{\prime}\right)^{\prime} u+q(x)|u|^{2}=\bar{\lambda} w(x)|u|^{2},
$$


respectively. Subtracting the two equations yields,

$$
-\left(p(x) u^{\prime}\right)^{\prime} \bar{u}+\left(p(x) \bar{u}^{\prime}\right)^{\prime} u=(\lambda-\bar{\lambda}) w(x)|u|^{2} .
$$

Integrating over $[a, b]$ by parts and applying the boundary conditions (1.2)-(1.3) yields

$$
0=(\lambda-\bar{\lambda}) \int_{a}^{b} w(x)|u|^{2} d x
$$

Since $\lambda$ is not real we have $\lambda-\bar{\lambda} \neq 0$, hence

$$
\int_{a}^{b} w(x)|u|^{2} d x=0 .
$$

Hence the result.

Next we state without proof a very important theorem in ordinary differential equations. The interested reader can refer to [12] for a proof of the theorem.

Theorem 2.0.13. (An existence and uniqueness theorem for a linear differential equation) Consider the equation

$$
p_{0}(x) u^{(n)}+p_{1}(x) u^{(n-1)}+\ldots+p_{n-1}(x) u^{\prime}+p_{n}(x) u=v(x) .
$$

If $p_{0}(x), p_{1}(x), \ldots, p_{n}(x)$ and $v(x)$ are (piecewise) continuous functions of $x$ in the interval $a \leq x \leq b$ and $p_{0}(x)$ does not vanish at any point on that interval, the differential equation admits a unique solution which, together with its first $(n-1)$ derivatives, is continuous in $(a, b)$ and satisfies the following initial conditions: $u\left(x_{0}\right)=u_{0}, u^{\prime}\left(x_{0}\right)=u_{0}^{\prime}, \ldots, u^{(n-1)}\left(x_{0}\right)=$ $u_{0}^{(n-1)}$ where $x_{0}$ is a point of $(a, b)$ and the $u_{0}^{(j)}$ are given.

It therefore follows from Theorem 2.0.13 that (1.1)-(1.2) has one and only one continuous solution with a continuous derivative. The existence and uniqueness of solutions of (1.1)-(1.2) is covered in detail in Chapter 2 of [19].

\subsection{The Prüfer system}

We now consider a very useful tool in the study of Sturm-Liouville problems. The problem is recast as a first order system so as to permit easy access to results regarding the behavior of 
the solutions and their eigenvalues. For simplicity, we first consider the differential equation having the form

$$
\left(P(x) u^{\prime}(x)\right)^{\prime}+Q(x) u(x)=0, a<x<b,
$$

where $0<P(x), P^{\prime}(x)$ and $Q(x)$ are continuous on the interval $[a, b]$ and on the appropriate side at the endpoints. When dealing with Sturm-Liouville problems we are interested in asking and answering the following questions among many others:

1. How often does a solution oscillate in an interval $a<x<b$; i.e, how many zeros does it have?

2. How many maxima and minima does it have between a pair of consecutive zeros?

3. What happens to these zeros when one changes $P(x)$ and $Q(x)$ ?

These questions can be answered by first applying the Prüfer substitution

$$
u(x)=r(x) \sin \theta(x)
$$

where $r(x)>0$ and

$$
P(x) u^{\prime}(x)=r(x) \cos \theta(x)
$$

to the quantities in (2.3). This is done by introducing the new dependent variables $r(x)$ and $\theta(x)$ induced by the transformation above.

When $p u^{\prime}=0$, then $\cos \theta=0$ (since $r>0$ ) and so

$$
\theta=\frac{(2 n+1) \pi}{2}, \text { with } n \geq 0, \text { where } n \text { is an integer }
$$

and

$$
r^{2}=u^{2}>0
$$

When $p u^{\prime} \neq 0$ we see that

$$
r^{2}=u^{2}+P^{2} u^{2}
$$

so that

$$
\tan \theta(x)=\frac{u(x)}{P(x) u^{\prime}(x)}
$$

For any non-trivial solution we always have $r>0$ (because if $r(x)=0$, i.e., $u^{\prime}(x)=0=$ $u(x)$ for some particular $x$, then by the uniqueness theorem for second order linear ordinary differential equations $u(x)=0$ for all $x$, i.e., we have the trivial solution.) Differentiating (2.4) and (2.5) with respect to $x$ and using (2.3) we find the Prüfer system

$$
\theta^{\prime}=Q(x) \sin ^{2} \theta+\frac{1}{P(x)} \cos ^{2} \theta
$$




$$
r^{\prime}=\frac{1}{2}\left[\frac{1}{P(x)}-Q(x)\right] r \sin 2 \theta .
$$

The system is solved with initial data

$$
u(a)=r_{0} \sin \theta_{0}, \quad p(a) u^{\prime}(a)=r_{0} \cos \theta_{0} .
$$

Equations (2.6) and (2.7) give Prüfer's differential equations for the phase and amplitude respectively. Solving the Prüfer equations (2.6) and (2.7) is thus equivalent to solving the originally given equation (2.3). Any solution to the Prüfer system determines a unique solution to (2.3), and conversely.

The statement is justified by the theorem below.

Theorem 2.1.1. Let (2.3), (2.6) and (2.7) hold.

1. Suppose $\theta$ and $r$ are solutions of (2.6) and (2.7), respectively. Then $u=r \sin \theta$ is $a$ solution of (2.3) on $[a, b]$ and $P u^{\prime}=r \cos \theta$.

2. Suppose $u$ is a nontrivial solution of (2.3). Then there exists a solution $\theta$ of (2.6) and a solution $r$ of (2.7) satisfying $r(x) \neq 0$ for $x \in[a, b]$, such that $u=r \sin \theta$ and $P u^{\prime}=r \cos \theta$.

The proof of the above theorem is given in [19]. This complete equivalence of the two systems means that we can use whichever is more convenient. The advantage of the Prüfer system for the study of the zeros of $u$ are that

1. $u=0$ whenever $\theta$ is a multiple of $\pi$ and

2. the phase is a scalar function from which one directly constructs the solution $u(x, \lambda)$. The differential equation (2.6) is of order one in $\theta$ alone and satisfies a Lipschitz condition with Lipschitz constant

$$
L=\sup _{x \in[a, b]}\left|\frac{\partial F}{\partial \theta}\right| \leq \sup _{x \in[a, b]}|Q(x)|+\sup _{x \in[a, b]} \frac{1}{|P(x)|}
$$

where $L$ is finite in any closed interval in which $P$ and $Q$ are continuous. Moreover, $\cos ^{2} \theta$ and $\sin ^{2} \theta$ are uniformly bounded. This ensures the application of an existence and uniqueness theorem for nonlinear systems so that (2.6) has a solution over any interval on which $P>0$ and $P, Q$ are piecewise continuous functions (Picard's Theorem). Since the right-hand side of (2.6) is differentiable in $\theta$, it follows that (2.6) has a unique solution $\theta(x)$ for any initial 
value $\theta(a)=\gamma$. Once the unique solution of (2.6) is obtained, one can easily obtain a unique solution to (2.7) because the equation becomes separable. In fact, the solution to (2.7) is of the form:

$$
r=D \exp \left\{\frac{1}{2} \int_{0}^{x}\left(\frac{1}{P(t)}-Q(t)\right) \sin 2 \theta(t) d t\right\}
$$

where $D$ is an arbitrary nonzero constant. The integral is convergent because $\frac{1}{P}, Q$ are assumed integrable, while the trigonometric functions are uniformly bounded functions of the continuous function $\theta$, hence integrable.

The fact that the zeros of a solution $u(x)$ of (2.3) occur where the Prüfer phase $\theta$ has the values $n \pi$ leads to the phase, $\theta(x)$ being an increasing function of $x$ at these points. This is because $\sin \theta=0$ implies $\cos ^{2} \theta=1$. This yields $\theta^{\prime}(x)=\frac{1}{P}>0$; hence, in the plane spanned by $P u^{\prime}$ and $u$, the curve $\left(P(x) u^{\prime}(x), u(x)\right)$ crosses the horizontal $P u^{\prime}$-axis when $\theta=n \pi$ only in a counterclockwise sense as $x$ increases.

Lemma 2.1.2. If $Q(x)>0$, then $u(x)$ has exactly one maximum (or minimum) between two successive zeros of a given solution. Thus, an infinite sequence of maxima and minima above (or below) the $x$-axis is impossible if $Q(x)>0$ in $[a, b]$.

Proof. At a maximum (or minimum) of $u$, one has $0=P u^{\prime}=r \cos \theta \Leftrightarrow \cos \theta=0$, that is, $\sin ^{2} \theta=1$ and so a maximum (or minimum) of $u$ is located at those points where $\theta=\left(n+\frac{1}{2} \pi\right)$. But (2.6) implies that

$$
\theta^{\prime}=Q \sin ^{2} \theta=Q>0
$$

at these points. Consequently, $\theta(x)$ can cross the line $\theta=\left(n+\frac{1}{2} \pi\right)$ only once. If it has to cross it a second time as shown in Figure 2.1, the slope would have to be negative at the second crossing point, thus violating the inequality (2.8). Hence the result.

Theorem 2.1.3. Every solution $u(t)$ of (2.3) satisfies the inequality

$$
|u(x)| \leq K \exp \left[\frac{1}{2} \int_{a}^{x}\left|\frac{1}{P(s)}-Q(s)\right| d s\right]
$$

where $x \in(a, b)$ and $K=\sqrt{u^{2}(a)+\left(P(a) u^{\prime}(a)\right)^{2}}$. Moreover, if

$$
\int_{a}^{b}\left|\frac{1}{P(s)}-Q(s)\right| d s<\infty
$$

then every solution of (2.3) is (uniformly) bounded. 


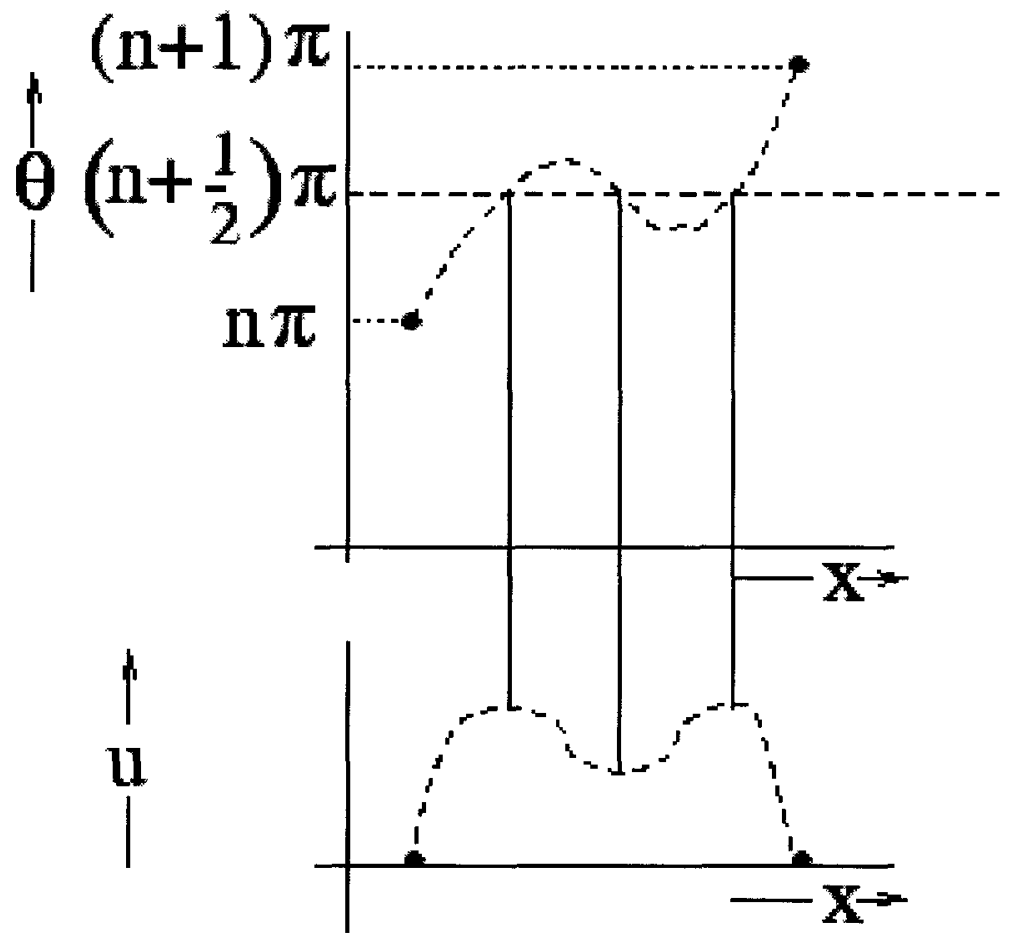

Figure 2.1: The minimum of $u(x)$ in this figure is not possible because the corresponding slope $\theta^{\prime}(x)$ at that point would have to be negative, violating inequality (2.8) 
The proof is given in [9]. We now apply the Prüfer transformation to equation (1.1) by setting

$$
\begin{aligned}
& P(x)=p(x) \\
& Q(x)=\lambda w(x)-q(x) .
\end{aligned}
$$

From (2.5) and (1.1) we get

$$
\theta_{\lambda}=\frac{\left[\left(p u^{\prime}\right) u_{\lambda}-\left(p u^{\prime}\right)_{\lambda} u\right]}{\left[u^{2}+\left(p u^{\prime}\right)^{2}\right]} \quad\left({ }_{\lambda}=\frac{\partial}{\partial \lambda}\right)
$$

Lemma 2.1.4. The numerator in (2.11) is equal to $\int_{a}^{x} w u^{2} d t$ for any real solution $u$ and for all $x$ satisfying $a<x \leq b$.

Proof. It is known that, for each $x$, the solutions $u(x, \lambda)$ depend continuously on the parameter $\lambda$ (in fact, they are an entire function of $\lambda,[4]$.)

From (1.1) and the implicit function theorem we see therefore that $\theta$ is also a differentiable function of $\lambda$ and

$$
\theta_{\lambda}=\frac{\partial}{\partial \lambda}\left[\left(-p u^{\prime}\right)^{\prime}+q u\right]=\frac{\partial}{\partial \lambda}[\lambda w u]
$$

This implies

$$
\left(\left(-p u^{\prime}\right)^{\prime}\right)_{\lambda}+q u_{\lambda}=\lambda w u_{\lambda}+w u
$$

Multiplying (1.1) by $u_{\lambda}$ and (2.12) by $u$ and subtracting the resulting equations we get

$$
\left(p u^{\prime}\right)^{\prime} u_{\lambda}-\left(p u^{\prime}\right)_{\lambda}^{\prime} u=w u^{2} .
$$

Integrating both sides of (2.13) over $[a, b]$, gives

$$
\int_{a}^{x} w u^{2} d t=\left[u_{\lambda} p u^{\prime}-\left(p u^{\prime}\right)_{\lambda} u\right]_{a}^{x}+\int_{a}^{x}\left[\left(p u^{\prime}\right)_{\lambda} u^{\prime}-p u^{\prime} u_{\lambda}^{\prime}\right] d t
$$

However,

$$
\begin{aligned}
\left(p u^{\prime}\right)_{\lambda} & =\frac{\partial\left(p u^{\prime}\right)}{\partial \lambda} \\
& =p \frac{\partial u^{\prime}}{\partial \lambda}, \text { since } p \text { is independent of } \lambda \\
& =p u_{\lambda}^{\prime}, \text { since }\left(u^{\prime}\right)_{\lambda}=\left(u_{\lambda}\right)^{\prime} \text {, because } u \text { is entire in } \lambda \text { for each fixed } x
\end{aligned}
$$

and so,

$$
\int_{a}^{x}\left[\left(p u^{\prime}\right)_{\lambda} u^{\prime}-p u^{\prime} u_{\lambda}^{\prime}\right] d t=0
$$


Therefore, $\int_{a}^{x} w u^{2} d t=\left[u_{\lambda} p u^{\prime}-\left(p u^{\prime}\right)_{\lambda} u\right]_{a}^{x}$. The boundary condition (1.2) ensures that $\left(p u^{\prime}\right)(a) u_{\lambda}(a)-\left(p u^{\prime}\right)_{\lambda}(a) u(a)=0$. Hence the result.

From this we conclude that $\theta(x, \lambda)$ is an increasing function of $\lambda$ whenever $w>0$ for all $x$ satisfying $a<x \leq b$.

Lemma 2.1.5. (Lemma 1.1 in [16])

Let $\lambda, u(x, \lambda)$ be an eigenvalue-eigenfunction pair of (1.1)-(1.3) with $\lambda$ real or non real and where we assume, more generally, that $q, r \in L(a, b)$. Then a necessary and sufficient condition that $\lambda$ be non simple is that

$$
\int_{a}^{b} u^{2}(x, \lambda) w(x) d x=0 .
$$

Proposition 2.1.6 (Richardson, [18]). For the problem (1.1)-(1.3), if $|\lambda|$ is sufficiently large, the number of zeros of the eigenfunction associated with two successive eigenvalues differs by precisely one.

The proof of this proposition was given by Atkinson and Jabon in [7], among others. They further give the following definitions of the Richardson numbers:

$$
\lambda^{+}=\inf \left\{x \in \Re \mid \forall \lambda>x, \theta_{\lambda}(b, \lambda)>0\right\},
$$

and

$$
\lambda^{-}=\sup \left\{x \in \Re \mid \forall \lambda<x, \theta_{\lambda}(b, \lambda)<0\right\} .
$$

That is, $\lambda^{+}$is the smallest real number such that all real eigenvalues greater than $\lambda^{+}$have eigenfunctions that behave as in a typical Sturm-Liouville problem, i.e., each eigenvalue is uniquely associated with its oscillation number. We note that if $w(x)>0$, for all $x \in[a, b]$, then $\lambda^{+}=-\infty$. If $w(x)$ changes sign on $[a, b]$ and $T$ is positive definite, then $\lambda^{+}=\lambda^{-}=0$. In the non-definite case, where $w(x)$ is generally sign indefinite as is $T$, the determination of these numbers is a very significant and difficult problem. We shall try to determine the two numbers for the problem at hand, in the next chapter but focus our attention to $\lambda^{+}$only (the other case being similar).

Lemma 2.1.7. A sufficient condition that the solutions of (2.3) should have at least $m$ zeros in $(a, b)$ is that

$$
\frac{Q}{P} \geq \frac{m^{2} \pi^{2}}{(b-a)^{2}}
$$

For the proof of this result, the interested reader can refer to [12]. We verify this result using the problem in the following example. 


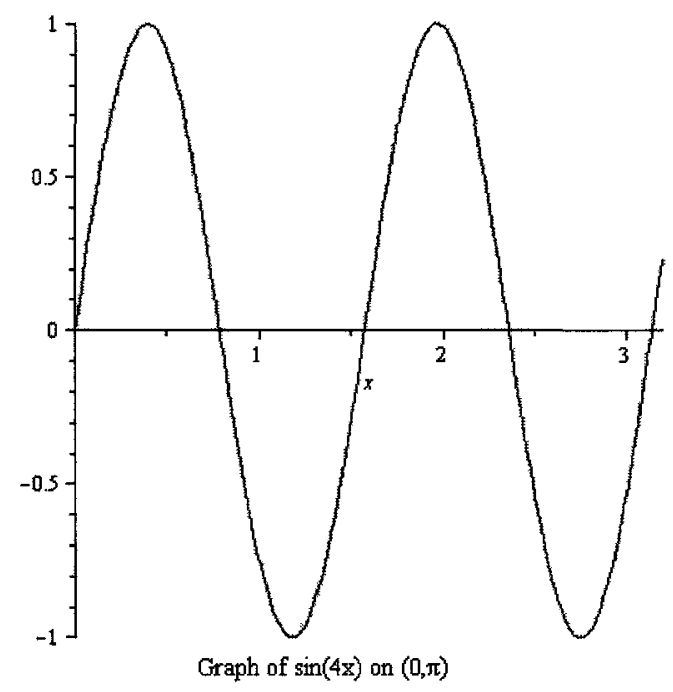

Figure 2.2: Graph of $\sin (4 x)$

Example 2.1.8. Given the boundary problem

$$
u^{\prime \prime}+\lambda u=0 \quad u(0)=0=u(\pi)
$$

we find its eigenfunctions to be

$$
u_{n}(x)=A_{n} \sin (n x) \quad n \geq 1,
$$

where the constants $A_{n}$ are arbitrary but nonzero. Without loss of generality we can therefore take $A_{n}=1$ for all $n$. In the problem, $Q=\lambda$ and $P=1, a=0$ and $b=\pi$. The sufficient condition that the differential equation should possess a solution with at least four zeros in $(0, \pi)$ is that $\lambda \geq 16$. However, for $\lambda=16$ we have $u_{4}(x)=\sin 4 x$ which has exactly three zeros in $(0, \pi)$. The statement is only true for $\lambda>16$. This shows that the lemma is not generally true because it does not hold for all equations. In this particular case, the lemma holds only when the inequality changes to

$$
\frac{Q}{P}>\frac{m^{2} \pi^{2}}{(b-a)^{2}}
$$

The graph in Figure 2.2 shows the number of zeros of $\sin 4 x$. 


\subsection{Comparison Theorems}

A good number of the corollaries, propositions and theorems below are stated and proved in [13]. In some cases proofs have been given and are taken from the same book.

Theorem 2.2.1. Suppose $F$ and $G$ are defined and continuous in a region $\Omega \subset \Re^{2}$ and $F(x, y) \geq G(x, y)$ there, and suppose further that each satisfies a Lipschitz condition with respect to $y$ on $\Omega$, with Lipschitz constant $L$. If there are functions $y(x)$ and $z(x)$ such that $y^{\prime}=F(x, y)$ and $z^{\prime}=G(x, z)$ on $(a, b)$ and if further $y(a) \geq z(a)$, then $y(x) \geq z(x)$ on $[a, b]$.

We can apply this result to the $\theta$ equation of the Prüfer system (2.6)-(2.7). Consider the initial-value problems

$$
\begin{aligned}
& \theta_{1}^{\prime}=Q_{1} \sin ^{2} \theta+\frac{1}{P_{1}} \cos ^{2} \theta \equiv F(x, \theta), \quad \theta_{1}(a)=\eta_{1} . \\
& \theta_{2}^{\prime}=Q_{2} \sin ^{2} \theta+\frac{1}{P_{2}} \cos ^{2} \theta \equiv G(x, \theta), \quad \theta_{2}(a)=\eta_{2} .
\end{aligned}
$$

Here the region $\Omega=[a, b] \times \Re$ and the coefficients all satisfy the conditions in (1.1)

Proposition 2.2.2. Suppose that in (2.15)-(2.16) $\quad Q_{2} \geq Q_{1}$ and $0<P_{2} \leq P_{1}$. If $\eta_{2} \geq \eta_{1}$ then $\theta_{2}(x) \geq \theta_{1}(x)$ on $[a, b]$.

Proof. The assumptions mean that $F(x, \theta) \geq G(x, \theta)$ and $F$ and $G$ satisfy a Lipschitz condition with Lipschitz constant

$$
L=\sup _{x \in[a, b]}\left|\frac{\partial F}{\partial \theta}\right| \leq \sup _{x \in[a, b]}\left|Q_{1}(x)\right|+\sup _{x \in[a, b]} \frac{1}{\left|P_{1}(x)\right|}
$$

A similar estimate holds for $G$. The constant $L$ is finite in any closed interval in which $Q_{i}$ and $P_{i}$ with $i=1,2$ are continuous as is the case with the problem at hand. Then applying Theorem 2.2.1, we see that $\theta_{2}(x) \geq \theta_{1}(x)$ on $[a, b]$.

Corollary 2.2.3. Suppose that one of the assumptions of Proposition 2.2.2, is strengthened: $Q_{1}<Q_{2}$ on $(a, b)$. Then $\theta_{2}(x)>\theta_{1}(x)$ on $(a, b]$.

Theorem 2.2.4. (Sturm Comparison Theorem)

Let $u_{1}$ and $u_{2}$ be nontrivial solutions of the differential equations

$$
\begin{aligned}
& \left(P_{1}(x) u_{1}^{\prime}(x)\right)^{\prime}+Q_{1}(x) u_{1}(x)=0 \\
& \left(P_{2}(x) u_{2}^{\prime}(x)\right)^{\prime}+Q_{2}(x) u_{2}(x)=0
\end{aligned}
$$


respectively where $u_{1}$ vanishes at points $x_{1}$ and $x_{2}$ in $[a, b]$. Suppose that $Q_{2}<Q_{1}$ and $(0<) P_{2}<P_{1}$ on $\left[x_{1}, x_{2}\right]$. Then, $u_{2}$ vanishes at least once on this interval, i.e, between any two zeros of $u_{1}$ there lies at least one zero of $u_{2}$. If $u_{1}$ has $k$ zeros in this interval, then $u_{2}$ has at least $k-1$ zeros there.

For the proof of this theorem, we refer the interested reader to [8].

Theorem 2.2.5. (Sturm-Picone theorem)

Suppose

$$
\begin{array}{r}
\left(P_{1} z^{\prime}\right)^{\prime}+Q_{1} z=0 \\
\left(P_{2} y^{\prime}\right)^{\prime}+Q_{2} y=0 \\
z(a)=z(b)=0
\end{array}
$$

where $Q_{2} \geq Q_{1}, 0<P_{1} \leq P_{2}$ on $[a, b]$, and equality does not hold everywhere on $[a, b]$. Then $y(x)$ has a zero in $(a, b)$.

Theorem 2.2.6. (Sturm Separation Theorem)

If $u(t)$ and $v(t)$ are linearly independent real solutions on $(a, b)$ of (2.3), then their zeros separate each other on the interval $(a, b)$.

We need to obtain the Prüfer phase equation corresponding to (1.1)-(1.2) to get

$$
\theta^{\prime}=(\lambda w-q) \sin ^{2} \theta+\frac{1}{p} \cos ^{2} \theta, \quad \theta(a)=\gamma \in[0, \pi),
$$

where $\gamma=\arctan \left(\frac{-\alpha_{2}}{\alpha_{1}}\right)$ is obtained from the relation:

$$
\theta(a)=\arctan \frac{u(a)}{\left(p u^{\prime}\right)(a)} .
$$

The boundary conditions (1.2)-(1.3) yield

$$
u(a)=-\frac{\alpha_{2}\left(p u^{\prime}\right)(a)}{\alpha_{1}}
$$

for $\alpha_{1} \neq 0$. Substituting $u(a)$ gives the desired result. When $\alpha_{1}=0$, then $\theta(a)=\frac{\pi}{2}$.

Lemma 2.2.7. The solution $\theta(x, \lambda)$ of the initial-value problem (2.17) is for each fixed $x \in(a, b]$, a continuous, monotonically increasing function of $\lambda$.

Proof. We see that the functions $P, Q$ take the values $P_{1}=p_{1}, Q_{1}=\lambda_{1} w-q, P_{2}=p_{1}, Q_{2}=$ $\lambda_{2} w-q$ where $w(t)>0$. So if $\lambda_{2}>\lambda_{1}$, then $Q_{2}>Q_{1}$ since $w>0$ on $[a, b]$ and Corollary 2.2.3 implies that $\theta\left(x, \lambda_{2}\right)>\theta\left(x, \lambda_{1}\right)$ for any $x>a$. In other words, for any $x>a, \theta$ is a strictly increasing function of $\lambda$, whenever $w(t)>0$. 
Lemma 2.2.8. Suppose that for some $x_{n}>a, \theta\left(x_{n}, \lambda\right)=n \pi$, where $n$ is an integer. Then $\theta(x, \lambda)>n \pi$ for all $x>x_{n}$.

Proof. The result follows from the fact that, for each $\lambda$, the phase $\theta(x, \lambda)$ is a strictly increasing function of $x$ whenever $\theta$ is a multiple of $\pi$.

For any regular Sturm-Liouville problem that consists of the differential equation (1.1) and separated endpoint conditions (1.2)-(1.3), there exists an infinite sequence of eigenvalues (and so corresponding eigenfunctions) having no finite point of accumulation. In light of this, we state the following theorem as given in [8]. For the proof, the interested reader should refer to the same reference.

Theorem 2.2.9. (Sturm Oscillation Theorem)

The boundary-value problem (1.1)-(1.3) with $w(t)>0$ has an infinite sequence of eigenvalues $\left\{\lambda_{n}\right\}_{0}^{\infty}$ with $\lambda_{n}<\lambda_{n+1}$ and $\lambda_{n} \rightarrow+\infty$ as $n \rightarrow \infty$. The eigenvalues are labeled in such a way that an eigenfunction $u_{n}$ corresponding to $\lambda_{n}$ has precisely $n$ zeros in the open interval $(a, b)$.

The theorem can be verified by using the example below:

Example 2.2.10. Consider the problem below. We need to find the eigenvalues and corresponding eigenfunctions and plot their graphs to view how many interior zeros each has on the interval [1,4].

$$
\begin{gathered}
x^{2} u^{\prime \prime}+x u^{\prime}+\lambda u=0 \\
u^{\prime}(1)=0=u^{\prime}(4) .
\end{gathered}
$$

The equation above, can be written in Sturm-Liouville form as

$$
\left(x u^{\prime}\right)^{\prime}+\frac{\lambda}{x} u=0
$$

Here, $p(x)=x>0, w(x)=\frac{1}{x}>0$ on $[1,4]$, and $q(x)=0$. Solving this equation, we find that the eigenvalues are $\lambda_{n}=\frac{n^{2} \pi^{2}}{(\ln 4)^{2}}, n \geq 0$ where $n$ is an integer, and the corresponding eigenfunctions are $u_{n}(x)=A_{n} \cos \left(\frac{n \pi}{\ln (4)} \ln (x)\right)$, where the constants $A_{n}$ are arbitrary and nonzero, so there is no loss of generality in assuming that $A_{n}=1$ and $u_{n}(x)=\cos \left(\frac{n \pi}{\ln (4)} \ln (x)\right), n \geq 0$. Then:

1. $\lambda_{0}=0, u_{0}(x)=1$, and has no zero on $[1,4]$.

2. $\lambda_{1}=\frac{\pi^{2}}{\ln (4)^{2}}, u_{1}(x)=\cos \left(\frac{\pi}{\ln (4)} \ln (x)\right)$ and has one interior zero on [1,4]. 
3. $\lambda_{2}=\frac{4 \pi^{2}}{\ln (4)^{2}}, u_{2}(x)=\cos \left(\frac{2 \pi}{\ln (4)} \ln (x)\right)$ and has two interior zeros on [1,4].

4. $\lambda_{3}=\frac{9 \pi^{2}}{\ln (4)^{2}}, u_{3}(x)=\cos \left(\frac{3 \pi}{\ln (4)} \ln (x)\right)$ and has three interior zeros on $[1,4]$.

5. $\lambda_{4}=\frac{16 \pi^{2}}{\ln (4)^{2}}, u_{4}(x)=\cos \left(\frac{4 \pi}{\ln (4)} \ln (x)\right)$ and has four interior zeros on [1,4].

6. $\lambda_{5}=\frac{25 \pi^{2}}{\ln (4)^{2}}, u_{5}(x)=\cos \left(\frac{5 \pi}{\ln (4)} \ln (x)\right)$ and has five interior zeros on [1,4].

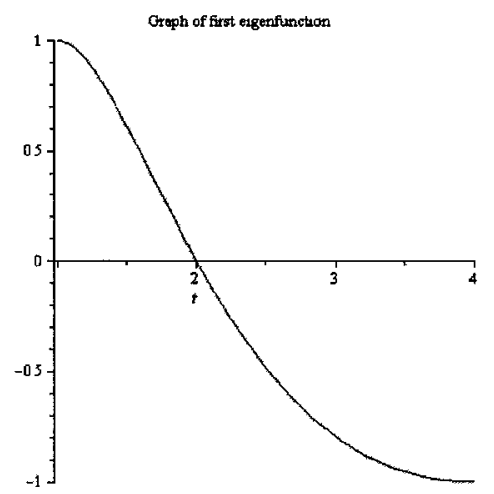

(a) Graph of $u_{1}(x)$

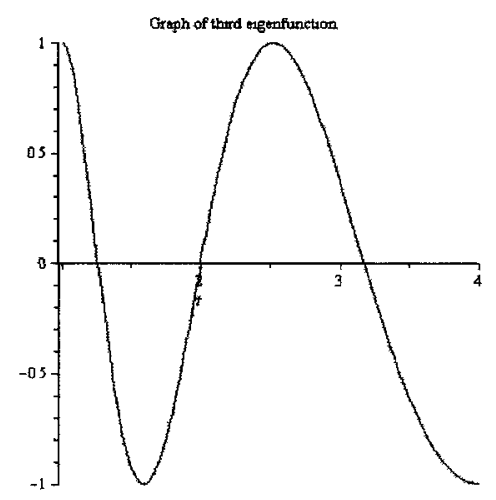

(c) Graph of $u_{3}(x)$

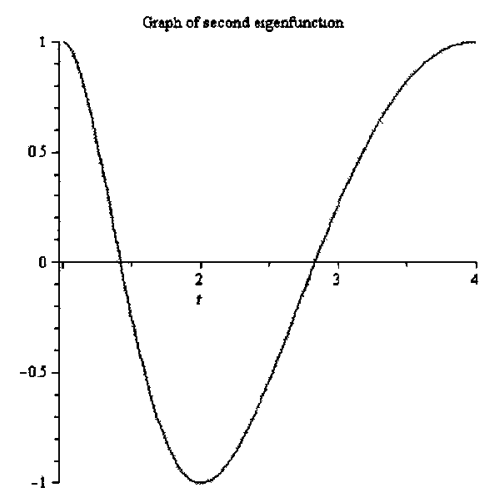

(b) Graph of $u_{2}(x)$

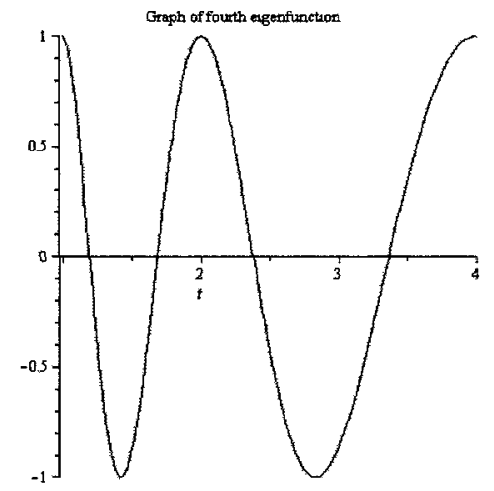

(d) Graph of $u_{4}(x)$

Figure 2.3: Graphs of the first four eigenfunctions of problem in Example 2.2.10

The results are shown in Figure 2.3.

The following two theorems are taken from [9]. For the proofs we refer the interested reader to the same reference.

Theorem 2.2.11. (Disconjugacy)

Consider the problem (2.3), where $x \in[a, \infty)$, and $u(a)=0$. Without loss of generallity we 
may assume that $u^{\prime}(a)>0$. If $P(x), Q(x)$ are continuous in $[a, \infty)$ and

$$
\int_{a}^{\infty}\left(\frac{1}{P(x)}+|Q(x)|\right) d x \leq \pi
$$

with $P(x)>0$, then no nontrivial solution of (2.3) has two zeros in $[a, \infty)$.

Theorem 2.2.12. Consider the problem (2.3) again with $u(a)=0,\left(u^{\prime}(a)>0\right)$, and $Q(x)<0$, for all $t \in[a, \infty)$. Then the nontrivial solution of $(2.3)$ has at most one zero in $[a, \infty)$. 


\section{Chapter 3}

\section{The Non-Definite Sturm-Liouville Problem}

We now apply the various results discussed in Chapter 2 to the specific problem

$$
u^{\prime \prime}(x)+(\lambda w(x)+q(x)) u(x)=0
$$

where $q(x)=q_{0} \in \Re$ for all $x \in[\alpha, \beta]$ and $w(x)$ is a step-function with two turning points inside $[\alpha, \beta]$. We can now assume that the finite interval $[\alpha, \beta]$ is the interval $[-1,2]$ since we can always transform the interval into $[-1,2]$ via the change of independent variable

$$
x \rightarrow \frac{3 x-(\beta+2 \alpha)}{\beta-\alpha} .
$$

We will assume that this transformation is effected and the interval is thus the interval $[-1,2]$. The weight function $w$ is now a piecewise constant step-function described by the relations

$$
w(x)= \begin{cases}a, & \text { if } x \in[-1,0], \\ b, & \text { if } x \in(0,1], \\ c, & \text { if } x \in(1,2],\end{cases}
$$

with $a<0, b>0, c<0$ without loss of generality (observe that the case where $a>0, b<$ $0, c>0$ reduces to the previous one upon replacing $\lambda$ by $-\lambda$ and $w$ by $-w$ in (3.1).) In this work we consider the Dirichlet problem on $[-1,2]$ exclusively, given by the usual boundary conditions:

$$
u(-1)=0=u(2) .
$$

Let $H^{2}[-1,2]$ be the subspace of $L^{2}[-1,2]$ consisting of all continuously differentiable functions $u \in C^{\prime}[-1,2]$ such that $u^{\prime}$ is absolutely continuous on $[-1,2]$ and $u^{\prime \prime}+q(x) u \in$ 
$L^{2}[-1,2]$. Let $T$ be the linear operator in $L^{2}[-1,2]$ defined by

$$
\begin{aligned}
D(T) & =\left\{u \in H^{2}[-1,2] \mid u(-1)=0=u(2)\right\} \\
T u & =u^{\prime \prime}+q(x) u .
\end{aligned}
$$

Clearly $D(T)$ is dense in $L^{2}[-1,2]$ by Theorem 1 and for all $u, v \in D(T)$ we have

$$
\begin{aligned}
(T u, v) & =\int_{-1}^{2}\left(u^{\prime \prime}+q u\right) \bar{v} d x \\
& =\int_{-1}^{2} u^{\prime \prime} \bar{v} d x+\int_{-1}^{2} q u \bar{v} d x .
\end{aligned}
$$

Integrating the first term on the RHS by parts and applying boundary conditions (3.2) yields

$$
(T u, v)=-\int_{-1}^{2} u^{\prime} \overline{v^{\prime}} d x+\int_{-1}^{2} q(x) u \bar{v} d x
$$

Integrating the first term again yields

$$
\begin{aligned}
(T u, v) & =\int_{-1}^{2} u \overline{v^{\prime \prime}} d x+\int_{-1}^{2} q(x) u \bar{v} d x \\
& =\int_{-1}^{2} u\left(\overline{v^{\prime \prime}}+q(x) \bar{v}\right) d x \\
& =\int_{-1}^{2} u\left(\overline{\left.v^{\prime \prime}+q(x) v\right)} d x\right. \\
& =(u, T v) .
\end{aligned}
$$

This means that $T$ is symmetric and, since $D(T)$ is dense in $L^{2}[-1,2]$, its closure is selfadjoint.

Theorem 3.0.13. The form $(T u, u)$ arising from the operator $T$ defined by (3.3) is generally indefinite. 
Proof.

$$
\begin{aligned}
(T u, u) & =\int_{-1}^{2}\left(u^{\prime \prime}+q(x) u\right) \bar{u} d x \\
& =\int_{-1}^{2} u^{\prime \prime} \bar{u} d x+\int_{-1}^{2} q(x)|u|^{2} d x \\
& =-\int_{-1}^{2} u^{\prime} \overline{u^{\prime}} d x+\int_{-1}^{2} q(x)|u|^{2} d x \\
& =\int_{-1}^{2}\left(q(x)|u|^{2}-\left|u^{\prime}\right|^{2}\right) d x
\end{aligned}
$$

From the above result, we deduce that for $q \leq 0,(T u, u)<0$ for all $u \in D(T)$ which means that $-T \geq 0$. However, when $q>0$, we see that the form $(T u, u)$ may be sign-indefinite. By Sturm-Liouville theory we recall that there are always infinitely many eigenvalues having a fixed sign (positive or negative). Let us choose $q_{0}$ so that $T$ has both positive and negative eigenvalues. Then, it is easy to see that if we choose $u$ to be an eigenfunction corresponding to a positive eigenvalue of $T$ (defined by $(3.3)$ ) then $(T u, u)>0$. On the other hand, by assumption, since $T$ has a negative eigenvalue with eigenfunction $v$ then $(T v, v)<0$. So, fixing such a general value of $q$, there may exist functions $\mathrm{u}$ for which $(T u, u)>0$ and a possibly different set of $u$ 's for which $(T u, u)<0$ and so the operator $T$ is generally sign-indefinite. Similarly,

$$
\begin{aligned}
(w u, u) & =\int_{-1}^{2} w|u|^{2} d x \\
& =-|a| \int_{-1}^{0}|u|^{2} d x+b \int_{0}^{1}|u|^{2} d x-|c| \int_{1}^{2}|u|^{2} d x
\end{aligned}
$$

It is clear that the sign of $\lambda(w u, u)$ is indefinite since it generally depends on the relative sizes of $a, b, c$. In accordance with accepted terminology [18], the problem (3.1)-(3.2) is called non-definite whenever the forms $(T u, u)$ and $(w u, u)$ are each indefinite as to their signs for $u \in D(T)$. 
We verify inequality (2.9) using (3.1). For each fixed $\lambda$ and $q=q_{0}$,

$$
\begin{aligned}
\int_{-1}^{2}\left|\frac{1}{P(x)}-Q(x)\right| d x= & \int_{-1}^{2}\left|1-\lambda w(x)-q_{0}\right| d x \\
= & \int_{-1}^{0}\left|1-a \lambda-q_{0}\right| d x+\int_{0}^{1}\left|1-b \lambda-q_{0}\right| d x \\
& +\int_{1}^{2}\left|1-c \lambda-q_{0}\right| d x \\
= & \left|1-a \lambda-q_{0}\right|+\left|1-b \lambda-q_{0}\right|+\left|1-c \lambda-q_{0}\right|<\infty .
\end{aligned}
$$

Thus, by Theorem 2.1.3, the solutions of (3.1)-(3.2) are bounded on $[-1,2]$.

\subsection{Estimating the Richardson Numbers}

Next we determine how the zeros of the solution $u(x, \lambda)$ of problem (3.1)-(3.2) are affected when the parameter $\lambda$ is changed. We do this by applying Sturm's Comparison Theorem to the problem (3.1)-(3.2).

Let $\lambda_{1}$ and $\lambda_{2}$ be distinct (real) eigenvalues of the problem (3.1)-(3.2) such that $\lambda_{2}>\lambda_{1}$. We verify if it is true that between two zeros of the nontrivial solution $u\left(x, \lambda_{1}\right)$, there always lies a zero of $u\left(x, \lambda_{2}\right)$, for all $x \in[-1,2]$. Since $w(x)$ changes sign on $[-1,2]$ this is not an immediate consequence of the comparison theorem.

We compare the two different solutions using the comparison equations below (where we frequently omit the independent variable $x$ for convenience);

$$
\begin{gathered}
u_{1}^{\prime \prime}(x)+\left(\lambda_{1} w(x)+q(x)\right) u_{1}(x)=0 \\
u_{1}(-1)=0=u_{1}(2) \\
u_{2}^{\prime \prime}(x)+\left(\lambda_{2} w(x)+q(x)\right) u_{2}(x)=0 \\
u_{2}(-1)=0=u_{2}(2) .
\end{gathered}
$$

Then (see Theorem 2.2.5) we have $P_{1}(x)=P_{2}(x)=1>0$ and $Q_{1}(x)=\lambda_{1} w(x)+$ $q_{0}, Q_{2}(x)=\lambda_{2} w(x)+q_{0}$. Now, $w(x)$ is generally indefinite as to its sign so the inequality $Q_{1}(x)<Q_{2}(x)$ holds only for a certain range of values of $x$ whenever $\lambda_{2}>\lambda_{1}$. One such case is whenever we consider those values of $x$ for which $w(x)>0$. 


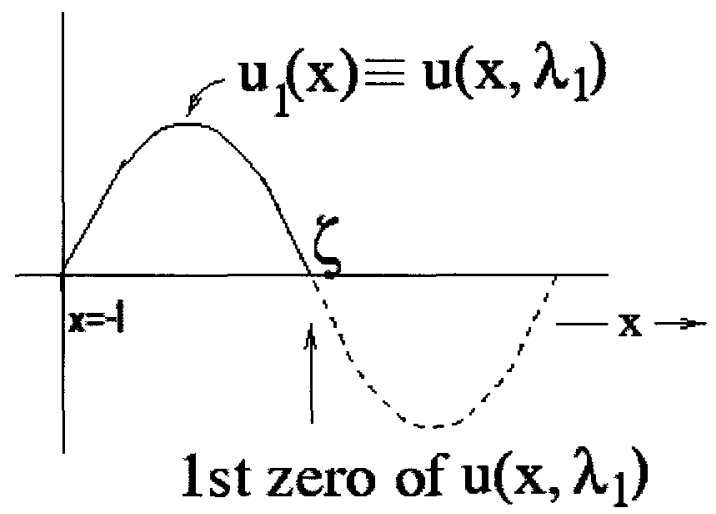

(a) solution $u\left(x, \lambda_{1}\right)$ with a zero at $\zeta$

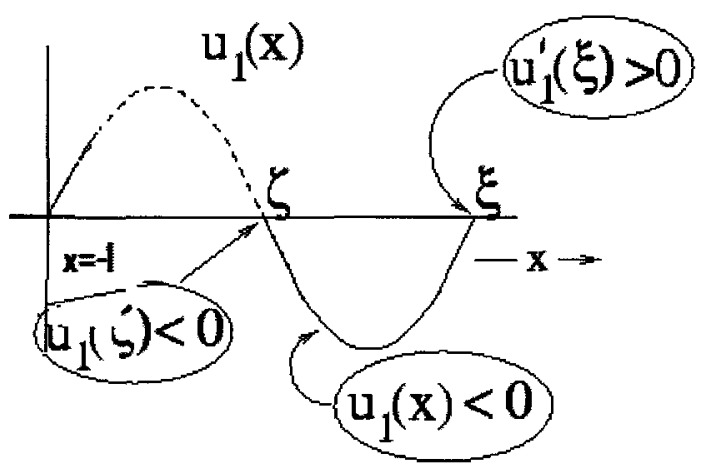

(b) solution $u\left(x, \lambda_{1}\right)$ with zeros at $\zeta$ and $\xi$

Figure 3.1: Graphs of a solution $u\left(x, \lambda_{1}\right)$

Multiply (3.4) and (3.5) by $u_{2}$ and $u_{1}$ respectively and then form their difference. The result, after canceling out the $q u_{1} u_{2}$ term is

$$
\left(u_{1}^{\prime \prime} u_{2}-u_{1} u_{2}^{\prime \prime}\right)=\left(\lambda_{2}-\lambda_{1}\right) u_{1} u_{2} w .
$$

Integrating, this gives

$$
\left[\left(u_{2} u_{1}^{\prime}-u_{1} u_{2}^{\prime}\right)\right]_{-1}^{x}=\left(\lambda_{2}-\lambda_{1}\right) \int_{-1}^{x} u_{1} u_{2} w d t .
$$

Applying the boundary conditions at $x=-1$, we have

$$
u_{2}(x) u_{1}^{\prime}(x)-u_{1}(x) u_{2}^{\prime}(x)=\left(\lambda_{2}-\lambda_{1}\right) \int_{-1}^{x} u_{1} u_{2} w d t .
$$

Now, assume that, for some range of values of $\lambda$, each of the corresponding solutions $u(x, \lambda)$ satisfying the boundary conditions at $x=-1$, oscillates. Certainly this must be true if $\lambda$ is sufficiently large, by Richardson's extension of Sturm-Liouville theory (see [18].) In other words, as $x$ increases beyond $x=-1, u(x, \lambda)$ reaches a maximum, then decreases, passes through a zero, reaches a minimum, increases and so on. Let $x=\zeta$ be the first zero of $u_{1}(x)$ as in Figure 3.1(a). Consequently,

$$
u_{2}(\zeta) u_{1}^{\prime}(\zeta)=\left(\lambda_{2}-\lambda_{1}\right) \int_{-1}^{\zeta} u_{1} u_{2} w d x
$$

We must now conclude that if $\lambda_{2}>\lambda_{1}$, then $u_{2}(x)$ has a zero in the interval $(0, \zeta)$. This is because for $\zeta>0$ then $u_{2}(x)$ must be zero inside the interval $(0, \zeta)$ by Sturm's comparison 
theorem. The situation on the interval $[-1,0]$ (and $[1,2])$ is a little different. The main reason is that the weight, $w(x)<0$ here and so the qualitative behavior of the solutions depends basically on the sign of $\lambda_{1} a+q_{0}$. For example, if $\lambda_{2}>\lambda_{1}>0$ then so long as $\lambda_{1} a+q_{0}<0$ we find that $\lambda_{2} a+q_{0}<\lambda_{1} a+q_{0}<0$ so that, by Sturm's comparison theorem again, $u_{2}(x)$ cannot have more than one zero in $[-1,0]$ (since $u_{1}(x)$ doesn't either). A similar argument applies to the case where $[-1,0]$ is replaced by $[1,2]$. It follows that for large values of $\lambda>0$ the zeros of $u(x)$ must accumulate in the intervals $[0,1]$ (see Figure 3.2).

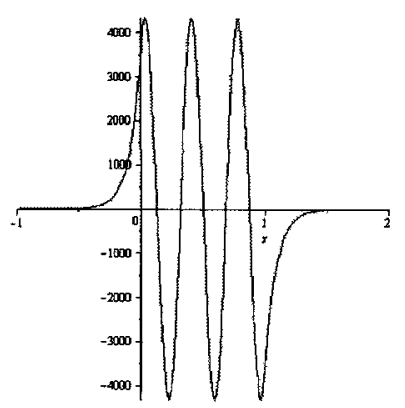

Figure 3.2: A typical solution $u(x)$ of $(3.1)$ on $[-1,2]$ for large $\lambda>0$.

Next, we turn to one of the main results of this thesis; the estimation of the Richardson numbers $\lambda^{+}$for the non-definite problem (3.1) - (3.2) in the case of a piecewise constant weight function having two turning points. We recall that the case of a piecewise constant weight function with one turning point was considered earlier in the important paper [7]. We recall the definition of these special numbers here for ease of use. In the next display of equations, $u(x, \lambda)$ is an eigenfunction of $(3.1)-(3.2)$ and

$$
\begin{aligned}
& \lambda^{+}=\inf \left\{x \in \Re: \forall \lambda>x, \int_{-1}^{2}|u(x, \lambda)|^{2} w(x) d x>0\right\} \\
& \lambda^{-}=\sup \left\{x \in \Re: \forall \lambda<x, \int_{-1}^{2}|u(x, \lambda)|^{2} w(x) d x<0\right\} .
\end{aligned}
$$

Note that these numbers $\lambda^{+}, \lambda^{-}$are generally distinct in the non-definite case due to the possible presence of ghost states (states for which the weighted square norm of the eigenfunction is zero). First we revisit the proof of Proposition 2 in [7] in the case of a piecewise constant weight function with one turning point and provide a corrected version since there is a serious typographical error there. In that important paper the authors considered a problem of the form (note the negative sign in front of the leading term)

$$
-y^{\prime \prime}+q y=\lambda w y, \quad y(-1)=y(1)=0,
$$




$$
\begin{gathered}
w(x)= \begin{cases}1, & \text { if } x \geq 0 \\
-1, & \text { if } x<0\end{cases} \\
q(x)=q_{0} \in \Re .
\end{gathered}
$$

They proceed to show that for (3.7), if $q_{0}<\frac{-\pi^{2}}{4}$, then $\lambda^{+} \leq\left|q_{0}\right|-\frac{\pi^{2}}{4}$. To prove the proposition, they applied two lemmas which we shall state and prove with some modifications to suit our needs.

Lemma 3.1.1. Let $y$ be a solution of $y^{\prime \prime}=-\mu y$ with $\mu \in \Re, x \in[-1,1]$.

1. If $\mu<\frac{\pi^{2}}{4}, y(0)=0$, and $y^{\prime}(x)>0$ in $[0,1]$, then

$$
\int_{0}^{1} y^{2}(x) d x<\frac{1}{2} y^{2}(1)
$$

2. If $\mu<\frac{\pi^{2}}{4}, y(-1)=0$, and $y^{\prime}(x)>0$ in $[-1,0]$, then

$$
\int_{-1}^{0} y^{2}(x) d x<\frac{1}{2} y^{2}(0)
$$

Proof. 1. We first consider the case $\mu \leq 0$. Since $y$ is increasing on $[0,1]$ and $y(0)=0$, this means that $y^{\prime \prime}=|\mu| y \geq 0$ in $[0,1]$. That is, $y$ is a convex function and by the theory of convex functions, if $g(x)$ is any line segment joining any two points on the curve $y(x)$, then $g(x) \geq y(x)$. If we let the two points be $(0, y(0))$ and $(1, y(1))$, then

$$
g(x)=x y(1) \text { and so } 0 \leq y(x) \leq x y(1) \text { for all } x \in[0,1]
$$

Thus,

$$
\int_{0}^{1} y^{2}(x) d x \leq \int_{0}^{1} x^{2} y^{2}(1) d x=\frac{1}{3} y^{2}(1)<\frac{1}{2} y^{2}(1) .
$$

as required. Next, we consider the case $0<\mu<\frac{\pi^{2}}{4}$. Then, necessarily,

$$
y(x)=C \sin k x, C>0,0<k<\frac{\pi}{2} \text { where } k^{2}=\mu .
$$

We then have,

$$
\int_{0}^{1} y^{2}(x) d x=C^{2} \int_{0}^{1} \sin ^{2} k x d x=\frac{C^{2}}{2}\left(1-\frac{\sin 2 k}{2 k}\right) .
$$


But for $0<k<\pi / 2$ we know that $\sin 2 k /(2 k)>\cos ^{2} k$. So,

$$
\frac{C^{2}}{2}\left(1-\frac{\sin 2 k}{2 k}\right)<\frac{C^{2}}{2}\left(1-\cos ^{2} k\right)=\frac{C^{2}}{2} \sin ^{2} k=\frac{y^{2}(1)}{2} \text {. }
$$

Therefore,

$$
\int_{0}^{1} y^{2}(x) d x<\frac{y^{2}(1)}{2}
$$

2. For the case $\mu \leq 0$, following the arguments in the proof of (1), we get

$$
0<y(x) \leq(x+1) y(0) \text { for all } x \in[-1,0]
$$

which yields

$$
\int_{-1}^{0} y^{2}(x) d x<\frac{y^{2}(0)}{2}
$$

Finally, if $0<\mu<\frac{\pi^{2}}{4}$, as in (1), we can take $y(x)=B \sin k(x+1), B>0$ and $k=\sqrt{\mu}$ is as defined in the proof of (1). Following the arguments in the proof of (1), we see that

$$
\int_{-1}^{0} y^{2}(x) d x<\frac{1}{2} y^{2}(0)
$$

Lemma 3.1.2. Let $y$ be a solution of $y^{\prime \prime}=-\mu y$ with $\mu \in \Re, x \in[-1,1]$.

1. If $\mu>0, y(1)=0$, and $y(0) y^{\prime}(0)>0$, then

$$
\int_{0}^{1} y^{2}(x) d x>\frac{1}{2} y^{2}(0)
$$

2. If $\mu>0, y(-1)=0$ and $y(0) y^{\prime}(0)<0$, then

$$
\int_{-1}^{0} y^{2}(x) d x>\frac{1}{2} y^{2}(0)
$$


Proof. 1. As before, we can write $y(x)=A \sin k(x-1)$, with $A \neq 0$, where $k=\sqrt{\mu}$. The condition $y(0) y^{\prime}(0)>0$ implies that $\sin 2 k<0$. So,

$$
\begin{aligned}
\int_{0}^{1} y^{2}(x) d x=A^{2} \int_{0}^{1} \sin ^{2} k(x-1) d x & =\frac{A^{2}}{2}\left(1-\frac{\sin 2 k}{2 k}\right) \\
& >\frac{A^{2}}{2}, \quad(\text { since } \sin 2 k<0) \\
& \geq \frac{A^{2}}{2} \sin ^{2} k=\frac{y^{2}(0)}{2} .
\end{aligned}
$$

2. We use an argument similar to the above, but instead use $y(x)=A \sin k(x+1)$. The condition $y(0) y^{\prime}(0)<0$ implies that $k \sin 2 k<0$ once again. A simple calculation as in the proof of (1) gives the result.

Remark: If $\mu>0, y(1)=0$, and $y(0) y^{\prime}(0) \geq 0$, then $\int_{0}^{1} y^{2}(x) d x \geq \frac{1}{2} y^{2}(0)$.

Proposition 3.1.3. (Proposition 2 in [7]) If in (3.7), $q_{0}<-\frac{\pi^{2}}{4}$, then $\lambda^{+} \leq\left|q_{0}\right|-\frac{\pi^{2}}{4}$.

We give a modified proof of the proposition, slightly different from the one given in [7], since there are a few misprints and errors in the paper that carry throughout the arguments.

Proof. Let $y$ satisfy (3.7), i.e, $y$ is an eigenfunction with eigenvalue $\lambda$ of the boundary problem

$$
\begin{aligned}
y^{\prime \prime}+\left(\lambda-q_{0}\right) y & =0, \quad 0 \leq x \leq 1 \\
y^{\prime \prime}+\left(-\lambda-q_{0}\right) y & =0, \quad-1 \leq x<0 \\
y(-1) & =y(1)=0 .
\end{aligned}
$$

Without loss of generality we may assume that $y^{\prime}(-1)=1$. Let $\lambda>\left|q_{0}\right|-\frac{\pi^{2}}{4}$. We will show that for such $\lambda$ we must have

$$
\int_{-1}^{1}|y(x, \lambda)|^{2} w(x) d x>0
$$


from which the stated result follows. Now, by assumption, $\lambda>-q_{0}-\frac{\pi^{2}}{4}$. So, $\mu=-\lambda-q_{0}<$ $\frac{\pi^{2}}{4}$. A moment's notice shows that we can now apply the second part of Lemma 3.1.1 to the interval $(-1,0)$ to find that (rewriting $y(x, \lambda) \equiv y(x)$ for simplicity)

$$
\int_{-1}^{0} y^{2}(x) d x<\frac{1}{2} y^{2}(0)
$$

Next, since by hypothesis we have $q_{0}<-\pi^{2} / 4$, it follows that $\left|q_{0}\right| \geq-q_{0}>\pi^{2} / 4$. So $\lambda$ defined at the outset must be positive. On the other hand, $\lambda>0$ and $q_{0}<-\pi^{2} / 4$ together imply that $\lambda-q_{0}>\lambda+\pi^{2} / 4>0$. Thus, if we consider (3.7) on $(0,1)$ then $\mu=\lambda-q_{0}>0$ here. In addition, it is easy to see that $k=\sqrt{\lambda-q_{0}}>\pi / 2$ since $\lambda>0$. Since $\lambda-q_{0}>0$ and since $y$ is increasing on $(-1,0)$, it follows that $y(0) y^{\prime}(0) \geq 0$. Hence, over $(0,1)$, we can apply the remark at the end of Lemma 3.1.2 to get

$$
\int_{0}^{1} y^{2}(x) d x \geq \frac{1}{2} y^{2}(0)
$$

Now, combining the above results, we obtain

$$
\begin{aligned}
\int_{-1}^{1} y^{2}(x) \operatorname{sgn}(x) d x & =-\int_{-1}^{0} y^{2}(x) d x+\int_{0}^{1} y^{2}(x) d x \\
& >-\frac{1}{2} y^{2}(0)+\frac{1}{2} y^{2}(0)=0
\end{aligned}
$$

Thus,

$$
\int_{-1}^{1} y^{2}(x) \operatorname{sgn}(x) d x>0, \text { for all } \lambda>\left|q_{0}\right|-\frac{\pi^{2}}{4}
$$

If $\theta(x, \lambda)$ is defined by

$$
\begin{gathered}
\theta(-1, \lambda)=0 \\
\tan \theta(x, \lambda)=\frac{y(x, \lambda)}{y^{\prime}(x, \lambda)},
\end{gathered}
$$

where $y(x, \lambda)$ is a nontrivial solution with $y(-1, \lambda)=0=y(1, \lambda)$, we have

$$
\frac{\partial \theta}{\partial \lambda}(1, \lambda)=\frac{1}{y^{2}+y^{\prime 2}} \int_{-1}^{1} y^{2}(t, \lambda) \operatorname{sgn}(t) d t>0, \quad \text { for all } \lambda>\left|q_{0}\right|-\frac{\pi^{2}}{4}
$$

Therefore, for eigenvalues $\lambda$ such that

$$
\lambda>\left|q_{0}\right|-\frac{\pi^{2}}{4}
$$


we have $\frac{\partial \theta}{\partial \lambda}>0$ and so it must be the case that (by definition),

$$
\lambda^{+} \leq\left|q_{0}\right|-\frac{\pi^{2}}{4} .
$$

Remark: By symmetry,

$$
\lambda^{-} \geq-\left|q_{0}\right|+\frac{\pi^{2}}{4}
$$

We now return to the problem under consideration, that of estimating the Richardson numbers of the problem (3.1) in the special case where we fix the values of $a, b$ and $c$ to $-1,2$ and -1 , respectively. Thus, (3.1) becomes

$$
u^{\prime \prime}(x)+(\lambda w(x)+q(x)) u(x)=0
$$

where $q(x)=q_{0} \in \Re, \quad \forall x \in[-1,2]$ and

$$
w(x)= \begin{cases}-1, & \text { if } x \in[-1,0] \\ 2, & \text { if } x \in(0,1], \\ -1, & \text { if } x \in(1,2],\end{cases}
$$

and $u$ satisfies the (Dirichlet) boundary conditions (3.2). We now estimate the value of $\lambda^{+}$(the estimation of $\lambda^{-}$is not undertaken here but can be done in a similar way).

Although we can prove lemmas similar in spirit to those of Atkinson-Jabon [7], it becomes very difficult to use those results to derive sharp estimates of the Richardson numbers in this case of two turning points in contrast with the one turning point case considered by these authors.

In [7], another approach to the problem of finding $\lambda^{+}$is the following, which is less precise, but slightly more general. The conditions on the weight-function $w$ and potential $q$ are quite general as mere Lebesgue integrability is assumed by the authors. For our purposes it suffices that each be piecewise continuous on $[a, b]$. In addition, we provide a complete yet different proof than the one sketched in [7].

Proposition 3.1.4. (Proposition 3 in [7])

Let $\lambda$ be a real eigenvalue and $y(x, \lambda)$ be a corresponding real eigenfunction of

$$
y^{\prime \prime}+(\lambda w+q) y=0, \quad y(a)=y(b)=0
$$

with zeros

$$
a=x_{0}<x_{1}<x_{2}<\cdots<x_{k}=b
$$


For each $j=0,1, \ldots, k-1$, let there be a number $\mu_{\jmath}<\lambda$ and a real valued function $u_{3}$ satisfying the differential equation

$$
u_{\jmath}^{\prime \prime}+\left(\mu_{\jmath} w+q\right) u_{\jmath}=0, \quad x \in\left[x_{\jmath}, x_{\jmath+1}\right]
$$

with the additional property that $u_{\jmath}(x)>0$ in $\left[x_{\jmath}, x_{\jmath+1}\right]$. Then, $\lambda^{+}<\lambda$; i.e., the Richardson number $\lambda^{+}$for (3.9) is less than the given eigenvalue $\lambda$.

Proof. Fix $j, 0 \leq \jmath \leq k-1$, and consider the function $z_{\jmath}=y / u_{\jmath}$ on $\left[x_{\jmath}, x_{\jmath+1}\right]$. A long but straightforward calculation shows that $z_{\jmath}$ satisfies a differential equation of the form

$$
-\left(P_{\jmath}(x) z_{\jmath}^{\prime}\right)^{\prime}+Q(x) z_{\jmath}=\left(\lambda-\mu_{\jmath}\right) W_{\jmath}(x) z_{\jmath}, \quad z_{\jmath}\left(x_{\jmath}\right)=z_{\jmath}\left(x_{\jmath+1}\right)=0,
$$

which is another Sturm-Liouville equation with leading term $P_{\jmath}=u_{\jmath}{ }^{2}>0$, potential term $Q=0$ and weight function $W_{\jmath}=w u_{\jmath}{ }^{2}$. This new boundary problem is a polar or left-definite problem [see Chapter 10.7, [12]], [17] and so, for each $j$, the quantity $\nu_{\jmath}=\mu_{\jmath}-\lambda$ is an eigenvalue of the above Dirichlet problem over $\left[x_{\jmath}, x_{\jmath+1}\right]$. A simple integration by parts now shows that, for each $j$, the eigenfunctions $z_{\jmath}$ necessarily satisfy

$$
\nu_{\jmath} \cdot \int_{x_{\jmath}}^{x_{\jmath}+1} z_{\jmath}^{2} W_{\jmath} d x>0 .
$$

Observe that, by hypothesis and by construction, the numbers $\nu_{3}$ must be a positive eigenvalue of the previous display. Since $W_{\jmath}(x)=w(x) u_{\jmath}(x)^{2}$ the eigenfunctions $z_{\jmath}$ corresponding to these $\nu_{\jmath}$ must satisfy

$$
\int_{x_{\jmath}}^{x_{j+1}} w u_{\jmath}^{2} z_{\jmath}^{2} d x>0
$$

that is,

$$
\int_{x_{j}}^{x_{j+1}} w y^{2} d x>0
$$

by definition of the $z_{j}$ above. Since this holds for every $j$ under consideration we can sum over all such $j$ to find that for our value of $\lambda$ its eigenfunction $y$ satisfies the relation

$$
\sum_{\jmath=0}^{k-1} \int_{x_{\jmath}}^{x_{\jmath+1}} w y^{2} d x=\int_{a}^{b} w y^{2} d x>0
$$

It follows by definition that $\lambda^{+}<\lambda$. 
Remark: If for each $j$ the $\lambda<\mu_{\mathrm{g}}$ then we can conclude that $\lambda^{-}>\lambda$ (the proof is similar with few changes and so is omitted.)

Specializing to the case where for some $c, d \in(a, b), w(x)>0$ in $(c, d)$, then we have the following proposition.

Proposition 3.1.5. Let $w, q$ be piecewise continuous and $w(x) \not \equiv 0$ on any subinterval of $[a, b]$. Assume further that $w(x)>0$ only in $(c, d) \subset(a, b)$. For some $\mu \in \Re$, let

$$
u^{\prime \prime}+(\mu w+q) u=0
$$

have a positive solution in $[a, e]$ where $e \in(d, b)$. In addition, for some $\lambda^{*}>\mu$, let there be a solution $y$ not identically zero with $y(a)=0$ having a zero in $(c, d)$. Then $\lambda^{+}<\lambda^{*}$.

Proof. Let $\lambda^{*}>\mu$, and let $y$ be the solution of $y^{\prime \prime}+\left(\lambda^{*} w+q\right) y=0$ satisfying $y(a)=0$, where we can always assume that $y^{\prime}(a)=1$, without loss of generality. For those zeros $x_{1}<x_{2}<\ldots$ of $y(x)$ that are in $(a, e)$, we can use the given value of $\mu$ to guarantee that there is a solution $u$ of (3.10) that is positive in $\left[x_{\jmath}, x_{\jmath+1}\right]$. This leaves the zeros of $y(x)$ in $(e, b)$. But $w(x)<0$ on $(e, b)$. Thus, we can always find a value of $\mu>0$ such that (3.10) is disconjugate on $[e, b]$. We can now apply Proposition 3.1.4 above to conclude that $\lambda^{+}<\lambda^{*}$.

We now apply Proposition 3.1.5 to the case at hand, given by (3.8) and its special coefficients.

Proposition 3.1.6. Let $w, q$ be as in Proposition 3.1.5 where, in addition,

$$
\begin{aligned}
\mu w+q & \leq 0, x \in(a, c), \\
\mu w+q & \geq 0, x \in(c, d), \\
\mu w+q & \leq 0, x \in(d, e), \\
(d-c) \sup _{(c, d)} \sqrt{\mu w+q} & \leq \frac{\pi}{2}, \\
(d-c) \inf _{(c, d)} \sqrt{\lambda^{*} w+q} & >\pi .
\end{aligned}
$$

Then $\lambda^{+}<\lambda^{*}$.

Proof. In $(a, c), \mu w+q \leq 0$ and so, by Theorem 2.2.5, there is a solution $u$ which is positive in $(a, c)$. On $(c, d)$, we compare the equations below:

$$
\begin{aligned}
u^{\prime \prime}+(\mu w+q) u & =0 \\
v^{\prime \prime}+\frac{\pi^{2}}{4(d-c)^{2}} v & =0, v(c)=0, v(d)=1 .
\end{aligned}
$$


We see that (3.12) has the solution $v(x)=\sin \left(\frac{\pi(x-c)}{2(d-c)}\right)$. It is also clear that $v(x) \neq 0$ in $(c, d]$. Therefore, (3.12) is disconjugate by Sturm-Liouville theory. Since $\mu w+q \leq \frac{\pi^{2}}{4(d-c)^{2}}$, we have that (3.11) is also disconjugate by Sturm's comparison theorem. Therefore, there exists a solution $u>0$ in $(c, d)$. In $(d, e), \mu w+q \leq 0$, and so the equation is disconjugate once again. So there is a positive solution in $(d, e)$. Therefore, we have established the fact that there is a solution of (3.10) that is positive on $[a, e]$. Now $(d-c) \inf _{(c, d)} \sqrt{\lambda^{*} w+q}>\pi$, means that

$$
\lambda^{*} w+q>\frac{\pi^{2}}{(d-c)^{2}} .
$$

Comparing the equations

$$
\begin{aligned}
y^{\prime \prime}+\left(\lambda^{*} w+q\right) y & =0 \\
v^{\prime \prime}+\frac{\pi^{2}}{(d-c)^{2}} v & =0, v(c)=v(d)=0
\end{aligned}
$$

we see that (3.15) has the solution $v(x)=\sin \left(\frac{\pi(x-c)}{d-c}\right)$ that vanishes at both endpoints of the interval. Inequality (3.13) implies that $y$ has at least one zero in $(c, d)$, by Sturm's comparison theorem. Therefore, Proposition 3.1.5 is satisfied and so $\lambda^{+}<\lambda^{*}$.

Now consider the particular case

$$
u^{\prime \prime}+(\lambda w(x)+q(x)) u=0, \quad u(-1)=u(2)=0,
$$

where $w(x)$ is the piecewise constant weight function defined by $w(x)=-1, x \in[-1,0)$, $w(x)=2, x \in[0,1)$ and $w(x)=-1, x \in(1,2]$, and $q$ is an arbitrary piecewise continuous function over $[-1,2]$. This is equivalent to the linked problem

$$
\begin{aligned}
u^{\prime \prime}+(-\lambda+q(x)) u & =0, x \in(-1,0) \\
u^{\prime \prime}+(2 \lambda+q(x)) u & =0, x \in(0,1) \\
u^{\prime \prime}+(-\lambda+q(x)) u & =0, x \in(1,2),
\end{aligned}
$$

since the same parameter $\lambda$ appears in each of the three equations.

Proposition 3.1.7. For some $M$, with $M>\frac{\pi^{2}}{20}$, we assume that

$$
\begin{array}{r}
q(x) \leq M x \in(-1,0) \\
-M \leq q(x) \leq M, x \in\left(0, \frac{\pi}{2 \sqrt{5 M}}\right) \\
q(x) \leq M x \in\left(1,1+\frac{\pi}{2 \sqrt{5 M}}\right) .
\end{array}
$$

Then, for (3.16), we have

$$
\lambda^{+}<\frac{21 M}{2}
$$


Proof. The results follow from Proposition 3.1.6, with the choice $\mu=2 M$. We consider, $a=-1, c=0, d=\frac{\pi}{2 \sqrt{5 M}}, e=1+\frac{\pi}{2 \sqrt{5 M}}, b=2$. Thus,

$$
\begin{aligned}
(d-c) \sup _{(c, d)} \sqrt{2 \mu+q(x)} & =\frac{\pi}{2 \sqrt{5 M}} \sup _{\left(0, \frac{\pi}{2 \sqrt{5 M}}\right)} \sqrt{2 \mu+q(x)} \\
& \leq \frac{\pi \sqrt{5 M}}{2 \sqrt{5 M}}=\frac{\pi}{2}, \\
(d-c) \inf _{(c, d)} \sqrt{2 \lambda^{*}+q(x)} & =\frac{\pi}{2 \sqrt{5 M}}\left(0, \frac{\pi}{2 \sqrt{5 M}}\right) \\
& \geq \frac{\pi \sqrt{2 \lambda^{*}-M}}{2 \sqrt{5 M}}, \\
& \left.>\frac{\pi \sqrt{20 M}}{2 \sqrt{5 M}} \text { (if } \lambda^{*}>21 M / 2\right) \\
& =\pi .
\end{aligned}
$$

Note that there are no bounds on $q(x)$ in $\left(\frac{\pi}{2 \sqrt{5 M}}, 1\right)$ and in $\left(1+\frac{\pi}{2 \sqrt{5 M}}, 2\right)$.

\subsection{The Nature of the Spectrum}

We state the following theorems without proof; they are meant to complement the thesis and bring the reader up to date on material herein. One should also consult the recently published book [6] where the discussion centers mainly on two-parameter Sturm-Liouville problems, including non-definite cases. The interested reader can also refer to [13].

Theorem 3.2.1. Consider the boundary value problem (3.1)-(3.2) in the non-definite case. Then there is a sequence of positive eigenvalues $\left\{\lambda_{k}^{+}\right\}_{k=0}^{\infty}$ for which $\lambda_{k}^{+} \rightarrow+\infty$ as $k \rightarrow \infty$ and a sequence of negative eigenvalues $\left\{\lambda_{k}^{-}\right\}_{k=0}^{\infty}$ for which $\lambda_{k}^{-} \rightarrow-\infty$ as $k \rightarrow \infty$.

This guarantees that in the non-definite case there are infinitely many positive and negative eigenvalues and possibly some non-real ones (called ghosts). There are results regarding the number of interior zeros of the eigenfunctions; for these, we can state that there is an integer $n_{R}$, called the Richardson index having the property that whenever $n \geq n_{R}$ there are exactly two eigenfunctions of (3.1)-(3.2) oscillating $n$-times in $(a, b)$. More information on this can be found in the survey paper [17] (updated references are in [6].)

In addition, in the non-definite case we know that the (positive) real eigenvalues may be labeled in such a way that whenever $\lambda=\lambda_{n}^{+}$its eigenfunction has $\mathrm{n}$ zeros in $(a, b)$ (a similar 
result is valid for the negative ones.) In this case, their asymptotic distribution is also known (see [5]) and given by

$$
\frac{\lambda_{n}^{+}}{n^{2}} \sim \frac{\pi^{2}}{\left(\int_{a}^{b} \sqrt{w_{+}(x)} d x\right)^{2}}, \quad n \rightarrow \infty,
$$

where $w_{+}(x)=\max \{w(x), 0\}$ is the positive part of $w(x)$.

The following results are taken from [2] where we refer the interested reader for the proofs.

Lemma 3.2.2. Let $\lambda \in C$ be an eigenvalue of (3.1)-(3.2) and $u(x, \lambda)$ be a corresponding eigenfunction. Then $\lambda$ is non-simple if and only if $(w u, \bar{u})=0$.

Lemma 3.2.3. Let $\lambda \in C, \operatorname{Im} \lambda \neq 0$ be a non-simple eigenvalue of (3.1)-(3.2). If $u(x, \lambda)=$ $u+i v$ is a corresponding eigenfunction, then

$$
\begin{gathered}
(w u, v)=(w u, u)=0 \\
(L u, u)=(L v, v)=0 \\
(L u, v)=0 .
\end{gathered}
$$

In [1], it is shown that (3.1) does not have a positive eigenfunction if it has a non-real eigenvalue or more generally, if it admits a ghost state. In [2], they give the following theorem related to this statement.

Theorem 3.2.4. (Theorem 2.0 (b.i) in [2])

If (3.1) admits a positive eigenfunction $u$ with eigenvalue $\lambda$, then there are no ghost states, except possibly u itself.

Theorem 3.2.5. Assume Thas one negative eigenvalue. Let $w \in L(a, b)$ be chosen so that (3.1) has a non-real eigenvalue. Then every non-real eigenvalue is simple. 


\section{Chapter 4}

\section{Explicit Solution of the Non-Definite Problem}

The piecewise smooth solution to problem $(3.1)-(3.2)$ is given by

$$
u(x)= \begin{cases}X(x), & \text { if } x \in[-1,0] \\ Y(x), & \text { if } x \in(0,1] \\ Z(x), & \text { if } x \in(1,2]\end{cases}
$$

where $a<0, b>0, c<0$, and

$$
\begin{gathered}
X(x)=\frac{\sin \left(\sqrt{-\lambda|a|+q_{0}}(x+1)\right)}{\sqrt{-\lambda|a|+q_{0}}} \\
Y(x)=\frac{\sqrt{\lambda b+q_{0}} \sin \left(\sqrt{-\lambda|a|+q_{0}}\right) \cos \left(\sqrt{\lambda b+q_{0}} x\right)}{\sqrt{-\lambda|a|+q_{0}} \sqrt{\lambda b+q_{0}}} \\
+\frac{\sqrt{-\lambda|a|+q_{0}} \cos \left(\sqrt{-\lambda|a|+q_{0}}\right) \sin \left(\sqrt{\lambda b+q_{0}} x\right)}{\sqrt{-\lambda|a|+q_{0}} \sqrt{\lambda b+q_{0}}}
\end{gathered}
$$




$$
\begin{aligned}
Z(x)= & \frac{\sin \left(\sqrt{-\lambda|a|+q_{0}}\right) \cos \left(\sqrt{\lambda b+q_{0}}\right) \cos \left(\sqrt{-\lambda|c|+q_{0}}(x-1)\right)}{\sqrt{-\lambda|a|+q_{0}}} \\
& +\frac{\cos \left(\sqrt{-\lambda|a|+q_{0}}\right) \sin \left(\sqrt{\lambda b+q_{0}}\right) \cos \left(\sqrt{-\lambda|c|+q_{0}}(x-1)\right)}{\sqrt{\lambda b+q_{0}}} \\
& +\frac{\cos \left(\sqrt{-\lambda|a|+q_{0}}\right) \cos \left(\sqrt{\lambda b+q_{0}}\right) \sin \left(\sqrt{-\lambda|c|+q_{0}}(x-1)\right)}{\sqrt{-\lambda|c|+q_{0}}} \\
& -\frac{\sqrt{\lambda|b|-q_{0}} \sin \left(\sqrt{-\lambda|a|+q_{0}}\right) \sin \left(\sqrt{\lambda b+q_{0}}\right) \sin \left(\sqrt{-\lambda|c|+q_{0}}(x-1)\right)}{\sqrt{-\lambda|a|+q_{0}} \sqrt{-\lambda|c|+q_{0}}} .
\end{aligned}
$$

The solution is found by piecing together the various solutions on the intervals $(-1,0),(0,1)$ and $(1,2)$ so as to obtain a continuously differentiable function on $(-1,2)$. As a result we find the eigenvalues by solving the dispersion relation for problem $(3.1)-(3.2)$, which is given by

$$
\begin{aligned}
0= & \sqrt{-\lambda|c|+q_{0}} \sqrt{\lambda b+q_{0}} \sin \left(\sqrt{-\lambda|a|+q_{0}}\right) \cos \left(\sqrt{\lambda b+q_{0}}\right) \cos \left(\sqrt{-\lambda|c|+q_{0}}\right) \\
& +\sqrt{-\lambda|c|+q_{0}} \sqrt{-\lambda|a|+q_{0}} \cos \left(\sqrt{-\lambda|a|+q_{0}}\right) \sin \left(\sqrt{\lambda b+q_{0}}\right) \cos \left(\sqrt{-\lambda|c|+q_{0}}\right) \\
& +\sqrt{-\lambda|a|+q_{0}} \sqrt{\lambda b+q_{0}} \cos \left(\sqrt{-\lambda|a|+q_{0}}\right) \cos \left(\sqrt{\lambda b+q_{0}}\right) \sin \left(\sqrt{-\lambda|c|+q_{0}}\right) \\
& -\left(\lambda b+q_{0}\right) \sin \left(\sqrt{-\lambda|a|+q_{0}}\right) \sin \left(\sqrt{\lambda b+q_{0}}\right) \sin \left(\sqrt{-\lambda|c|+q_{0}}\right) .
\end{aligned}
$$

We fixed the values of $a, b$ and $c$ to $-1,2$ and -1 respectively so that the dispersion relation is now a function of $\lambda$ for each value of $q_{0}$. We calculate a few non-real eigenvalues in the cases $q_{0}=\pi^{2}, 2 \pi^{2}, 3 \pi^{2}, 5 \pi^{2}$ and $6 \pi^{2}$ in the rectangle $D=\{\lambda \in C:|\Re \lambda|<300$ and $|\Im \lambda|<$ $300\}$ using the Maple package RootFinding[Analytic]. The graphs of the corresponding eigenfunctions for the cases $q_{0}=\pi^{2}$ and $q_{0}=6 \pi^{2}$ are given in Figures (4.1)-(4) and (4.3)(4.7), respectively. Properties of other non-real eigenfunctions for varying values of $q_{0}$ can be found in Tables 4.2-4.3.

In Table 4.1 we show that the positive eigenvalues obtained in the case where $q_{0}=6 \pi^{2}$ agree roughly with the asymptotic estimates of the Atkinson-Mingarelli Theorem (see Section 3.2 above). The eigenvalues are now labeled in accordance with the convention that the eigenvalue $\lambda_{n}$ has an eigenfunction with $n$ zeros in $(-1,2)$ (Haupt-Richardson Theorem [17], [18]). The asymptotic distribution of the eigenvalues then satisfies

$$
\frac{\lambda_{n}^{+}}{n^{2}} \sim \frac{\pi^{2}}{\left(\int_{-1}^{2} \sqrt{w_{+}(x)} d x\right)^{2}} \approx 4.9348, \quad n \rightarrow \infty .
$$

In this case, the Richardson index is 5 (see [17]) while our theoretical estimate for the Richardson number $\lambda^{+}<21 M / 2=621.785$ (Proposition 3.1.7.) Indeed, we see from the data here that $\lambda^{+} \leq 106.4765$. 


\begin{tabular}{|c||c|c|c||}
\hline$n$ & $\lambda_{n}$ & $\lambda_{n} / n^{2}$ & theoretical est. \\
\hline 5 & 106.4765 & 4.2591 & 4.9348 \\
\hline 6 & 166.6810 & 4.6300 & "' \\
\hline 7 & 235.7529 & 4.8113 & " \\
\hline 8 & 314.3121 & 4.9111 & " \\
\hline
\end{tabular}

Table 4.1: The first few positive eigenvalues in the case $q_{0}=6 \pi^{2}$.
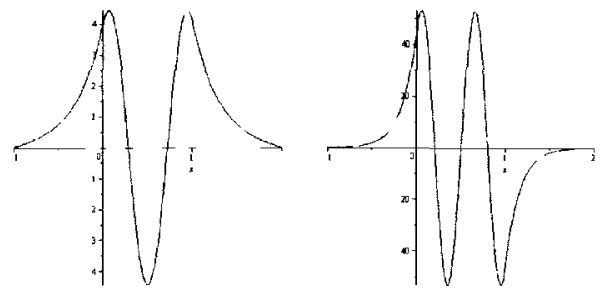

(a) $\lambda=20.588795$

(b) $\lambda=50.326686$
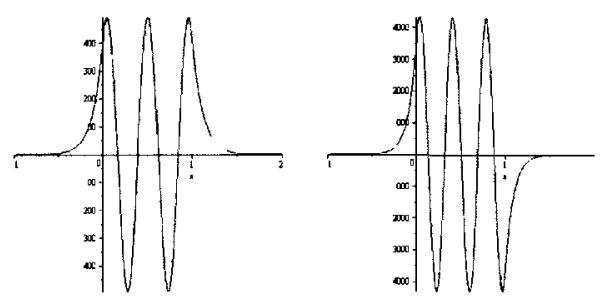

(c) $\lambda=89.154935$

(d) $\lambda=137.663115$

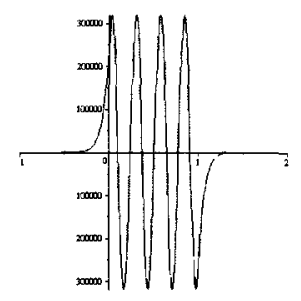

(e) $\lambda=264.082045$

Figure 4.1: Graphs of some eigenfunctions of $(3.1)-(3.2)$ for the case $q_{0}=\pi^{2}$ 


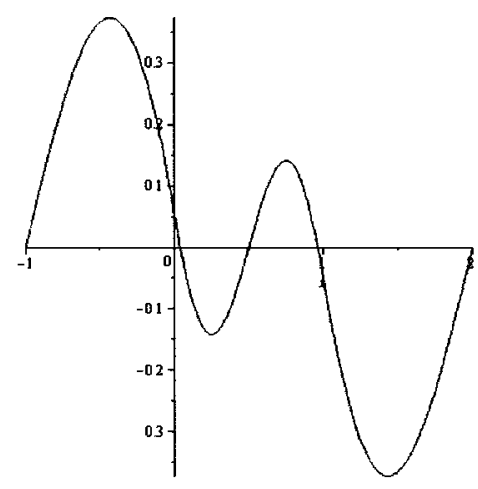

(a) Real Part

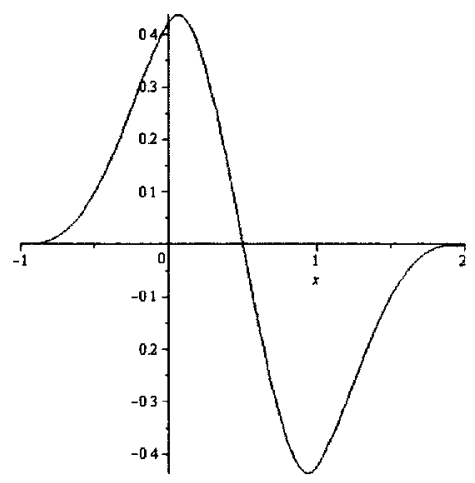

(b) Imaginary Part

Figure 4.2: Graphs of the real and imaginary part of the eigenfunction corresponding to $\lambda=3.2465+5.6334 i$ when $q_{0}=\pi^{2}$.

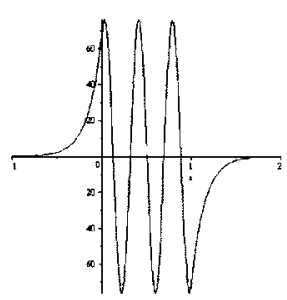

(a) $\lambda=106.476595$

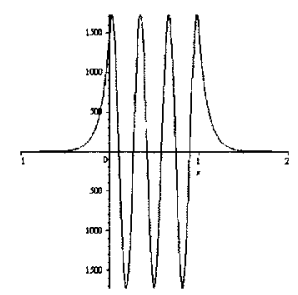

(b) $\lambda=166.681017$

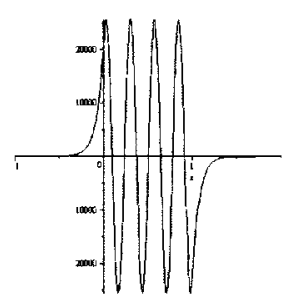

(c) $\lambda=235.752859$

Figure 4.3: Graphs of some eigenfunctions of $(3.1)-(3.2)$ for the case $q_{0}=6 \pi^{2}$

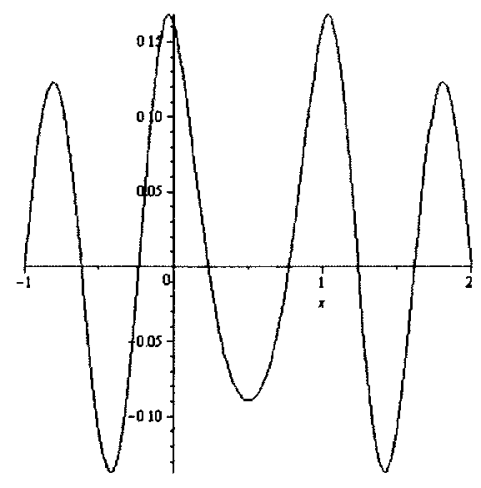

(a) Real Part

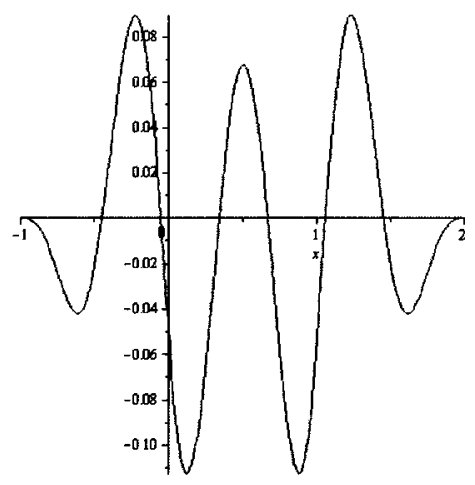

(b) Imaginary Part

Figure 4.4: Graphs of some eigenfunctions of $(3.1)-(3.2)$ for the case $q_{0}=6 \pi^{2}$ and $\lambda=-6.4344-14.4314 i$ 


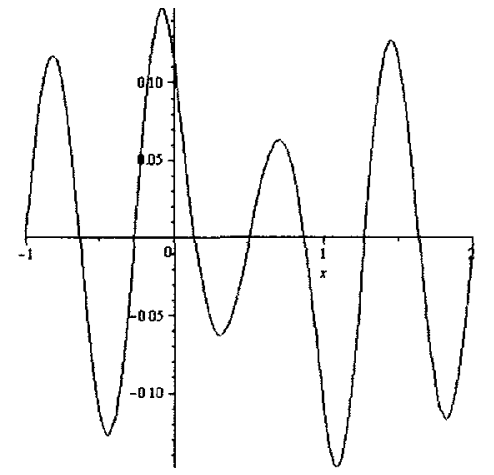

(a) Real Part

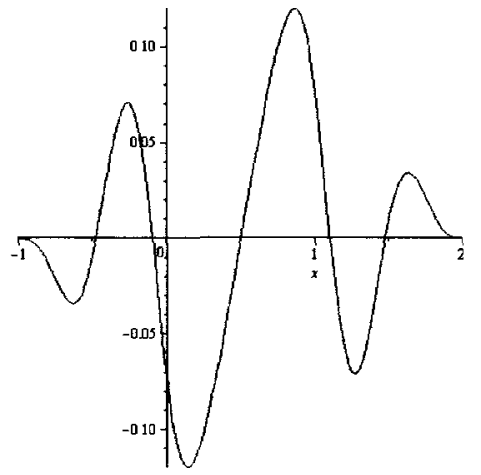

(b) Imaginary Part

Figure 4.5: Graphs of some eigenfunctions of $(3.1)-(3.2)$ for the case $q_{0}=6 \pi^{2}$ and $\lambda=-13.4034-13.5248 i$

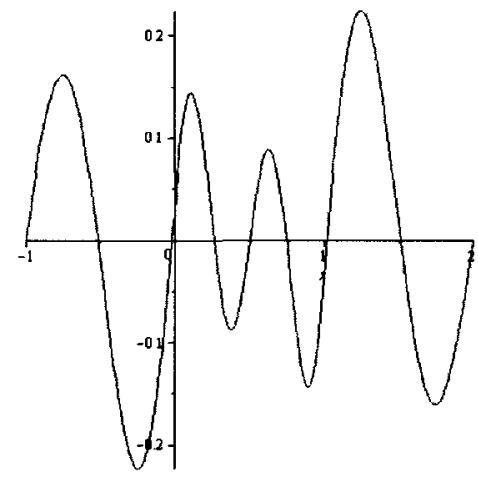

(a) Real Part

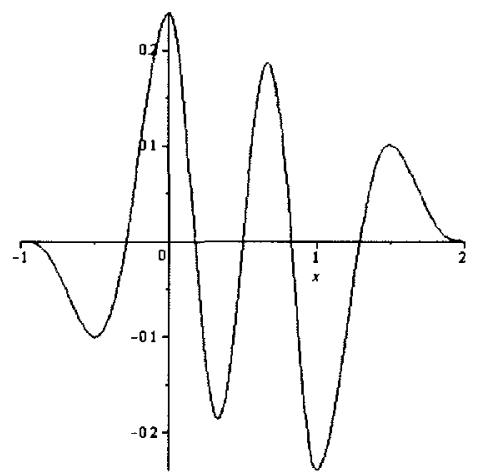

(b) Real Part

Figure 4.6: Graphs of some eigenfunctions of (3.1) - (3.2) for the case $q_{0}=6 \pi^{2}$ and $\lambda=21.5520-15.2468 i$ 


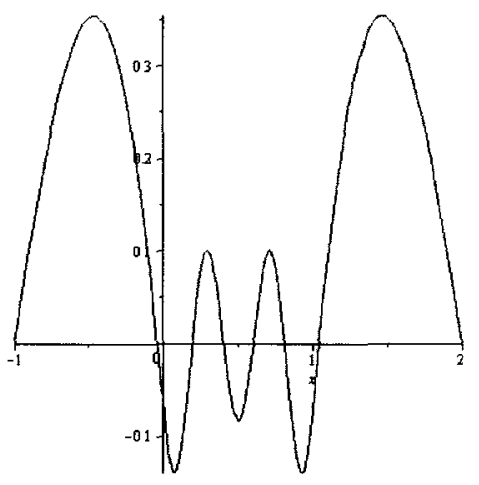

(a) Real Part

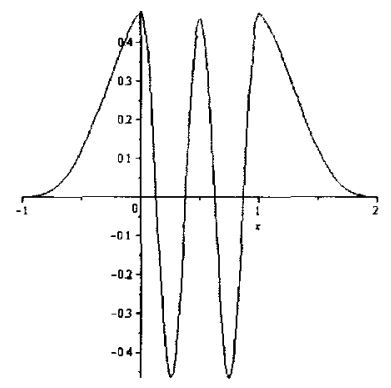

(b) Imaginary Part

Figure 4.7: Graphs of some eigenfunctions of $(3.1)-(3.2)$ for the case $q_{0}=6 \pi^{2}$ and $\lambda=52.0258+7.0997 i$ 


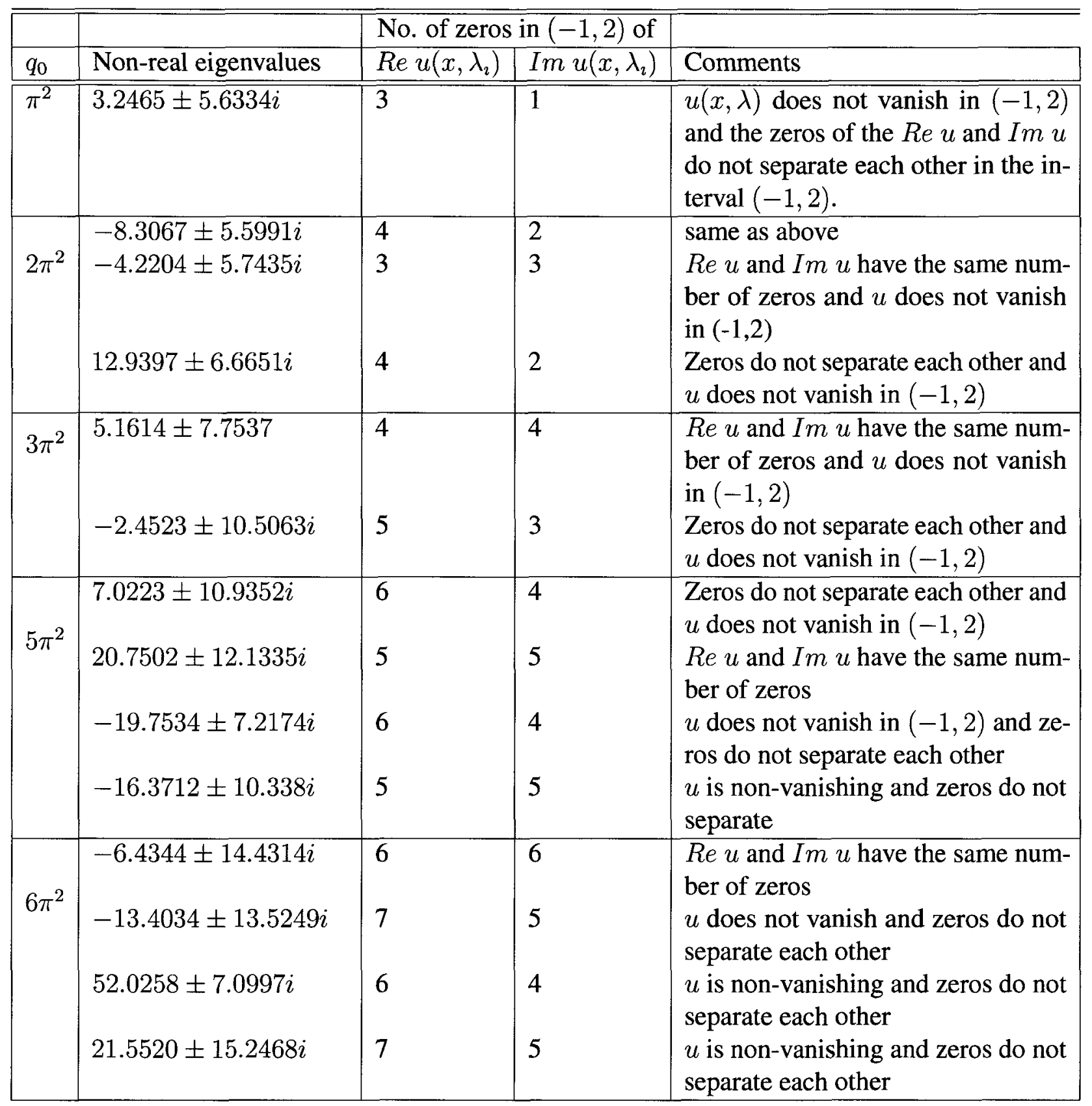

Table 4.2: Non-real eigenvalues obtained inside the rectangle $D$ for varying $q_{0}$ 


\begin{tabular}{|l|l|l|l|l|}
\hline \hline$q_{0}$ & $\begin{array}{l}\text { No. of complex } \\
\text { pairs of eigenval- } \\
\text { ues found }\end{array}$ & $\begin{array}{l}\text { No. of negative } \\
\text { eigenvalues con- } \\
\text { sidered }\end{array}$ & $\begin{array}{l}\text { total No. of eigen- } \\
\text { values considered }\end{array}$ & $\begin{array}{l}\text { Smallest oscilla- } \\
\text { tion number of } \\
\text { real eigenvalues } \\
\text { found }\end{array}$ \\
\hline \hline$\pi^{2}$ & 1 & 9 & 18 & 2 \\
\hline $2 \pi^{2}$ & 3 & 6 & 18 & 3 \\
\hline $3 \pi^{2}$ & 2 & 7 & 18 & 3 \\
\hline $5 \pi^{2}$ & 4 & 6 & 20 & 4 \\
\hline $6 \pi^{2}$ & 4 & 7 & 20 & 5 \\
\hline
\end{tabular}

Table 4.3: Summary of results of the spectrum of problem (3.1)-(3.2). In column 2, the number of non-real eigenvalues found may not be exact. 


\section{Chapter 5}

\section{Discussion}

The results shown in the figures and tables above are discussed here. Using Sturmian arguments we cannot have non-real eigenvalues for the cases where $q_{0} \leq 0$ and for the boundary conditions considered here. This is because the operator is left-definite or the Sturm-Liouville equation is in the orthogonal case, [17], [12]. We thus considered the cases where $q_{0}>0$ in particular, those where $q_{0}=\pi^{2}, 2 \pi^{2}, 3 \pi^{2}, 5 \pi^{2}$, and $6 \pi^{2}$. In [19], it is stated that for the case where $w(x)$ changes sign, the number of non-real eigenvalues is finite (see also [17] and the references therein where this is proved). It is shown in the same reference and in [16] that, when the leading second order term $p>0$ in (1.1), the number of non real eigenvalues is finite and an upper bound for this number is established. For most values of $q_{0}$ considered in this paper we have seen that there are both real and non-real eigenvalues. We see that the eigenvalues obtained in this case do not behave like those in the definite cases. For example, oscillation numbers can actually decrease as the parameter value increases, but then oscillations will eventually stabilize and the usual (Sturm) oscillation theorem may be claimed. The number of non-real eigenvalues also appears to increase with increasing $q_{0}$ as shown in Table 4 yet we do not know how to prove this in general. In fact, there are no such theorems known in this direction.

Table 4.3 gives a summary of the findings on the non-definite problem introduced in Chapter 3 . We see that the first three values of $q_{0}$ have exactly the same number of eigenvalues in the rectangle $D$ and the last two have the same number too. For all values of $q_{0}$ considered, there is none in which an eigenvalue has an oscillation number less than 2. However, for small positive values of $q_{0}$ there are cases where the eigenfunction corresponding to the first eigenvalue has no zero in $(-1,2)$ and is strictly positive in $(-1,2)$. These lead to the so-called definitizable cases of Allegretto and Mingarelli [2]. For example, the case $q_{0}=1.0966$ yielded $2.8072 \times 10^{-10}$ as the smallest eigenvalue in absolute value. The corresponding eigenfunction has no zero in $(-1,2)$ and is strictly positive there. The case $q_{0}=1.2$ has $\lambda=-0.1287$ as 
the smallest eigenvalue and its corresponding eigenfunction behaves in the same way. In both cases there are no non-real eigenvalues (and there cannot be any either; more on this below). However, in the cases where there are non-real eigenvalues, we have seen that there is no real eigenvalue whose eigenfunction is positive in $(-1,2)$ because the smallest oscillation number is 2. This verifies the results in [1] and shows the applicability of Theorem 3.2.4 in a two turning point case.

We have seen cases where four real eigenvalues (two of which are positive) have eigenfunctions with the same oscillation numbers. As the value of $q_{0}$ increases, the smallest oscillation number eventually increases too. One peculiar result is an observation based on the non-real eigenfunctions corresponding to non-real eigenvalues. We expect that the zeros of the real and imaginary parts of the non-real eigenfunctions should separate each other as is the case in the case of one turning point. In this non-definite case, Table 4.3 shows that the zeros do not separate each other. Instead, the difference between the number of zeros of the real and imaginary parts is two in some cases and zero in other cases which makes it impossible for the zeros to separate each other. Furthermore, the number of zeros of the imaginary part is less than or equal to that of the real part. Another peculiar result is that obtained in the case $q_{0}=6 \pi^{2}$. 


\section{Chapter 6}

\section{Conclusion}

Many thousands of papers by mathematicians and others, have been published on SturmLiouville problems since their origins in 1836 . Yet, remarkably, this subject is still an intensely active field of research today. In this thesis we undertook two projects: first, we gave a critical analysis of the important paper by Atkinson and Jabon [7] that led to the first major study of examples of Sturm-Liouville problems having non-real eigenvalues. This phenomenon was postulated in 1918 by Richardson in [18], with the first actual example found by Mingarelli [16]. We corrected some errors in [7] and presented different proofs of the results therein with an eye on the two turning point case. Secondly, we gave some theoretical estimates of the (positive) Richardson numbers of a Sturm-Liouville problem with fixed-end boundary conditions in the non-definite case where the weight function is piecewise constant and has two turning points.

We also undertook a numerical study of the non-real eigenfunctions and eigenvalues in this case, paralleling the study in [7] in the case of one turning point. Ultimately, our aim was to examine the behavior of the eigenfunctions, both real and non-real, of this non-definite Sturm-Liouville problem. One feature of the non-definite problem is the possible existence of non-real eigenvalues. This may sound paradoxical, as the equation is (formally) self-adjoint and so all eigenvalues should be real. However, this is where the problem lies: the formal selfadjointness of an equation does not necessarily imply the self-adjointness of the corresponding operator. It follows that whenever there is a non-real eigenvalue the corresponding operator cannot be self-adjoint in a Hilbert space.

We have indeed verified that if the problem (3.1)-(3.2) has at least one (complex conjugate) pair of non-real eigenvalues, then there is no real eigenvalue whose corresponding eigenfunction has one zero in the interval $(-1,2)$ (in conformity with the results in [2], [1]). We also showed 
that, in the cases considered here, the complex eigenfunctions (corresponding to non-real eigenvalues) are never zero in $(-1,2)$. Whether this is an accident or the result of a more general yet unproven theorem is unknown. For these same examples, we showed that the real and imaginary parts of these eigenfunctions do not have interlacing zeros. In fact, these zeros are expected to alternate in the one-turning-point case as shown by Richardson [18], but in the case of two turning points we see that there are examples where the zeros do not alternate at all. We also noticed that, in the examples under consideration, the number of zeros of the real part of each of the non-real eigenfunctions considered is greater than or equal to the number of zeros of the imaginary part. This may also be a consequence of a more general theorem, we don't know. In future studies on this subject there is the need for the formulation of a theorem that could explain some or all of these observations. Finally, we observed that even for real eigenvalues the corresponding eigenfunctions do not behave in conformity with the Sturm oscillation theorem as was postulated and proved by Haupt (1911) in [11], and Richardson [18]. 


\section{Bibliography}

[1] W. Allegretto and A. B. Mingarelli. On the non-existence of positive solution for a Schrödinger equation with an indefinite weight-function. C. R. Math. Rep. Acad. Sci. Canada, 8:69-72, 1986.

[2] W. Allegretto and A. B. Mingarelli. Boundary problems of the second order with an indefinite weight-function. J. Reine Angew. Math., 398:1-24, 1989.

[3] T. G. Anderson, R. C. Brown, and D. B. Hinton. Perturbation theory for a one-term weighted differential operator. In Spectral theory and computational methods of SturmLiouville problems (Knoxville, TN, 1996), volume 191 of Lecture Notes in Pure and Appl. Math., pages 149-170. Dekker, New York, 1997.

[4] F. V. Atkinson. Discrete and Continuous Boundary Problems. Academic Press, New York, London, 1964.

[5] F. V. Atkinson and A. B. Mingarelli. Asymptotics of the number of zeros and of the eigenvalues of general weighted Sturm-Liouville problems. J. Reine Angew. Math., 375/376:380-393, 1987.

[6] F. V. Atkinson and A. B. Mingarelli. Multiparameter Eigenvalue Problems (SturmLiouville Theory). CRC Press. Taylor \& Francis, New York, 2010.

[7] F.V Atkinson and D Jabon. Indefinite Sturn-Liouville problems. in Proc. 1984 workshop on Spectral Theory of Sturm-Liouville Differential Operators, pages 31-44. Argon National Laboratory, Argon,Illinois, 1984.

[8] Garrett Birkhoff and Gian-Carlo Rota. Ordinary differential equations. John Wiley \& Sons Inc., New York, fourth edition, 1989.

[9] George Chailos. Applications of Prüfer transformations in the theory of ordinary differential equations. Irish Math. Soc. Bull., (63):11-31, 2009.

[10] Lokenath Debnath and Piotr Mikusiński. Introduction to Hilbert spaces with applications. Academic Press Inc., Boston, MA, 1990. 
[11] O. Haupt. Untersuchungen über Oszillationstheoreme. Diss. Würzburg. Leipzig: B. Z. Teubner. 50 S. $8^{\circ}, 1911$.

[12] Edward Lindsay Ince. Ordinary Differential Equations. Dover, New York, 1956.

[13] Norman Lebovitz. Ordinary Differential Equations Textbook for Mathematics 27300. Chicago, 2010.

[14] John Locker. Functional analysis and two-point differential operators, volume 144 of Pitman Research Notes in Mathematics Series. Longman Scientific \& Technical, Harlow, 1986.

[15] M. A. Upali Mampitiya. Spectral asymptotics for polar vector Sturm-Liouville problems. C. R. Math. Rep. Acad. Sci. Canada, 7(6):381-386, 1985.

[16] A. B. Mingarelli. On the existence of nonsimple real eigenvalues for general SturmLiouville problems. Proc. Amer. Math. Soc., 89(3):457-460, 1983.

[17] Angelo B. Mingarelli. A survey of the regular weighted Sturm-Liouville problem-the nondefinite case. In International workshop on applied differential equations (Beijing, 1985), pages 109-137. World Sci. Publishing, Singapore, 1986.

[18] R. G. D. Richardson. Contributions to the Study of Oscillation Properties of the Solutions of Linear Differential Equations of the Second Order. Amer. J. Math., 40(3):283-316, 1918.

[19] Anton Zettl. Sturm-Liouville theory, volume 121 of Mathematical Surveys and Monographs. American Mathematical Society, Providence, RI, 2005. 